\title{
Dynamics of a two-dimensional competitive system of rational difference equations with quadratic terms
}

\author{
Vahidin Hadžiabdić ${ }^{1}$, Mustafa RS Kulenovićc ${ }^{*}$ and Esmir Pilav ${ }^{3}$
}

\section{"Correspondence:}

mkulenovic@mail.uri.edu

2Department of Mathematics,

University of Rhode Island, Kingston,

Rhode Island 02881-0816, USA

Full list of author information is

available at the end of the article

\section{Springer}

\begin{abstract}
We investigate global dynamics of the following systems of difference equations:

$$
\left\{\begin{array}{l}
x_{n+1}=\frac{b_{1} x_{n}^{2}}{A_{1}+y_{n}^{2}} \\
y_{n+1}=\frac{a_{2}+c_{2}^{2} y_{n}^{2}}{x_{n}^{2}},
\end{array} \quad n=0,1,2, \ldots\right.
$$

where the parameters $b_{1}, a_{2}, A_{1}, c_{2}$ are positive numbers and the initial condition $y_{0}$ is an arbitrary nonnegative number and $x_{0}$ is a positive number. We show that this system has rich dynamics which depends on the part of a parametric space. We find precisely the basins of attraction of all attractors including the points at $\infty$.

MSC: Primary 39A10; 39A30; secondary 37E99; 37D10
\end{abstract}

Keywords: basin of attraction; competitive map; global stable manifold; monotonicity; period-two solution

\section{Introduction}

In this paper we study the global dynamics of the following rational system of difference equations:

$$
\left\{\begin{array}{l}
x_{n+1}=\frac{b_{1} x_{n}^{2}}{A_{1}+y_{2}^{2}}, \\
y_{n+1}=\frac{a_{2}+c_{2} y_{n}^{2}}{x_{n}^{2}},
\end{array} \quad n=0,1,2, \ldots,\right.
$$

where the parameters $b_{1}, a_{2}, A_{1}, c_{2}$ are positive numbers and the initial condition $y_{0}$ is an arbitrary nonnegative number and $x_{0}$ is a positive number.

The related system of difference equations

$$
x_{n+1}=\frac{\beta_{1} x_{n}}{A_{1}+y_{n}}, \quad y_{n+1}=\frac{\alpha_{2}+\gamma_{2} y_{n}}{x_{n}}, \quad n=0,1, \ldots
$$

where the parameters $A_{1}, \beta_{1}, \alpha_{2}$ and $\gamma_{2}$ are positive numbers and the initial conditions $x_{0}>$ $0, y_{0} \geq 0$, was considered in [1], where it was shown that this system has simple dynamics. Precisely, it was shown that system (2) has no equilibrium points if $\beta_{1} \leq A_{1}$ and that it has a unique equilibrium point if $\beta_{1}>A_{1}$, in which case this equilibrium point is a saddle point. Furthermore, the following result describes the global dynamics of system (2). 
Theorem 1 Consider system (2).

(1) Assume that $\beta_{1}>A_{1}$ and $\gamma_{2} A_{1} \neq \alpha_{2}$. Then there exist a set $\mathcal{C} \subset \mathcal{R}$ which is invariant and a subset of the basin of attraction of $E$. The set $\mathcal{C}$ is a graph of a strictly increasing continuous function of the first variable on an interval (and so is a manifold) and separates $\mathcal{R}$ into two connected and invariant components, namely

$$
\mathcal{W}_{-}:=\left\{x \in \mathcal{R} \backslash \mathcal{C}: \exists y \in \mathcal{C} \text { with } x \preceq_{\text {se }} y\right\} \quad \text { and } \quad \mathcal{W}_{+}:=\left\{x \in \mathcal{R} \backslash \mathcal{C}: \exists y \in \mathcal{C} \text { with } y \preceq_{\text {se }} x\right\}
$$

which satisfy:

$$
\lim _{n \rightarrow \infty}\left(x_{n}, y_{n}\right)=(0, \infty) \quad \text { for every }\left(x_{0}, y_{0}\right) \in \mathcal{W}_{-}
$$

and

$$
\lim _{n \rightarrow \infty}\left(x_{n}, y_{n}\right)=(\infty, 0) \quad \text { for every }\left(x_{0}, y_{0}\right) \in \mathcal{W}_{+}
$$

(2) Assume that $\gamma_{2} A_{1}=\alpha_{2}$. Then system (2) can be decoupled as follows:

$$
x_{n+1}=\frac{\beta_{1} x_{n}^{2}}{A_{1} x_{n}+\beta_{1} \gamma_{2}}, \quad y_{n+1}=\frac{1}{\beta_{1}} y_{n}\left(A_{1}+y_{n}\right), \quad n=0,1, \ldots,
$$

and every solution of this system (depending of the choice of the initial condition $\left(x_{0}, y_{0}\right)$ ) is either bounded and converges to an equilibrium point, or increases monotonically to infinity.

(3) Assume that $\beta_{1} \leq A_{1}$ and $\gamma_{2} A_{1} \neq \alpha_{2}$. Every solution $\left\{\left(x_{n}, y_{n}\right)\right\}$ of system (2), with $x_{0}>0$, $y_{0} \geq 0$, satisfies

$$
\lim _{n \rightarrow \infty} x_{n}=0 \text { and } \quad \lim _{n \rightarrow \infty} y_{n}=\infty .
$$

Thus every solution of system (2) either converges to the unique equilibrium point or is asymptotic to one of the points at infinity, precisely to either $(0, \infty)$ or to $(\infty, 0)$. In all cases, either solution is eventually monotonic or the subsequences of even indexed and odd indexed terms are eventually monotonic. Introduction of quadratic terms into the system will substantially change the dynamics by introducing new equilibrium points (up to three) with different local character and minimal period-two solutions (up to 11). Again, most of the solutions of system (1) will be asymptotic to $(\infty, 0)$ or $(0, \infty)$, but the separatrix between the two basins of attraction may consist of several global stable manifolds of either saddle point equilibrium points or non-hyperbolic equilibrium points or minimal periodtwo solutions. In one case, when there exists a unique non-hyperbolic equilibrium point, it is possible that this point will have a basin of attraction of positive Lebesgue measure.

System (1) is a competitive system, and our results are based on recent results about competitive systems in the plane, see [2,3]. System (1) can be used as a mathematical model for competition in population dynamics. The first systematic study of a specific competitive system with quadratic terms was performed in [4] where system of the form

$$
x_{n+1}=\frac{x_{n}}{a+y_{n}^{2}}, \quad y_{n+1}=\frac{y_{n}}{b+x_{n}^{2}}, \quad n=0,1, \ldots,
$$


where the parameters $a, b>0$ and the initial conditions $x_{0}, y_{0} \geq 0$, was considered. It was shown that the dynamics of system (3) is very similar to the dynamics of the corresponding linear fractional system

$$
x_{n+1}=\frac{x_{n}}{a+y_{n}}, \quad y_{n+1}=\frac{y_{n}}{b+x_{n}}, \quad n=0,1, \ldots,
$$

with the same conditions on parameters and initial conditions. Both systems have nine parametric regions with different dynamical behavior.

As noted, the introduction of quadratic terms in system (2) dramatically changes the dynamics. The techniques used to study system (2) were straightforward calculations, while the techniques which will be used to study system (1) are a combination of techniques for studying real algebraic curves and implicit function theorem as neither equilibrium points nor period-two solutions are explicitly computable. Some of our calculations are performed by using Mathematica and outputs are included in the Appendix.

The paper is organized as follows. Section 2 contains some necessary results on competitive systems in the plane. Section 3 provides some basic facts about the equilibrium points and injectivity of the map associated with system (1). Section 4 contains local stability analysis of both equilibrium solutions and minimal period-two solutions. Section 5 gives global dynamics in different cases.

\section{Preliminaries}

A first-order system of difference equations

$$
\left\{\begin{array}{l}
x_{n+1}=f\left(x_{n}, y_{n}\right), \\
y_{n+1}=g\left(x_{n}, y_{n}\right),
\end{array} \quad n=0,1,2, \ldots,\right.
$$

where $\mathcal{S} \subset \mathbb{R}^{2},(f, g): \mathcal{S} \rightarrow \mathcal{S}, f, g$ are continuous functions, is competitive if $f(x, y)$ is non-decreasing in $x$ and non-increasing in $y$, and $g(x, y)$ is non-increasing in $x$ and nondecreasing in $y$. If both $f$ and $g$ are non-decreasing in $x$ and $y$, system (4) is cooperative. Competitive and cooperative maps are defined similarly. Strongly competitive systems of difference equations or strongly competitive maps are those for which the functions $f$ and $g$ are coordinate-wise strictly monotone. Competitive and cooperative systems have been investigated by many authors, see [2, 3, 5-14]. Special attention to discrete competitive and cooperative systems in the plane was given in $[2,3,5,6,9,10,14-23]$. One of the reasons for paying special attention to two-dimensional discrete competitive and cooperative systems is their applicability to mathematical models in biology and economics, the former involves competition or cooperation between two species. Another reason is that the theory of two-dimensional discrete competitive and cooperative systems is very well developed, unlike such theory for three and higher dimensional systems. Part of the reason for this situation is de Mottoni-Schiaffino theorem given below, which provides relatively simple scenarios for possible behavior of many two-dimensional discrete competitive and cooperative systems. However, this does not mean that one cannot encounter chaos in such systems as has been shown by Smith, see [14].

If $\mathbf{v}=(u, v) \in \mathbb{R}^{2}$, we denote by $\mathcal{Q}_{\ell}(\mathbf{v}), \ell \in\{1,2,3,4\}$ the four quadrants in $\mathbb{R}^{2}$ relative to $\mathbf{v}$, i.e., $\mathcal{Q}_{1}(\mathbf{v})=\left\{(x, y) \in \mathbb{R}^{2}: x \geq u, y \geq v\right\}, \mathcal{Q}_{2}(\mathbf{v})=\left\{(x, y) \in \mathbb{R}^{2}: x \leq u, y \geq v\right\}$, and so on. Define the south-east partial order $\preceq_{s e}$ on $\mathbb{R}^{2}$ by $(x, y) \preceq_{s e}(s, t)$ if and only if $x \leq s$ and $y \geq t$. 
Similarly, we define the north-east partial order $\preceq_{n e}$ on $\mathbb{R}^{2}$ by $(x, y) \preceq_{n e}(s, t)$ if and only if $x \leq s$ and $y \leq t$. For $\mathcal{A} \subset \mathbb{R}^{2}$ and $\mathrm{x} \in \mathbb{R}^{2}$, define the distance from $\mathrm{x}$ to $\mathcal{A}$ as $\operatorname{dist}(\mathrm{x}, \mathcal{A}):=$ $\inf \{\|\mathrm{x}-\mathrm{y}\|: \mathrm{y} \in \mathcal{A}\}$. By int $\mathcal{A}$ we denote the interior of a set $\mathcal{A}$.

It is easy to show that a map $F$ is competitive if it is non-decreasing with respect to the south-east partial order, that is, if the following holds:

$$
\left(\begin{array}{l}
x^{1} \\
y^{1}
\end{array}\right) \preceq_{s e}\left(\begin{array}{l}
x^{2} \\
y^{2}
\end{array}\right) \Rightarrow F\left(\begin{array}{l}
x^{1} \\
y^{1}
\end{array}\right) \preceq_{s e} F\left(\begin{array}{l}
x^{2} \\
y^{2}
\end{array}\right) .
$$

For standard definitions of attracting fixed point, saddle point, stable manifold, and related notions, see $[8,24]$.

We now state three results for competitive maps in the plane. The following definition is from [14].

Definition 1 Let $\mathcal{S}$ be a nonempty subset of $\mathbb{R}^{2}$. A competitive map $T: \mathcal{S} \rightarrow \mathcal{S}$ is said to satisfy condition $(O+)$ if for every $x, y$ in $\mathcal{S}, T(x) \preceq_{n e} T(y)$ implies $x \preceq_{n e} y$, and $T$ is said to satisfy condition $(O-)$ if for every $x, y$ in $\mathcal{S}, T(x) \preceq_{n e} T(y)$ implies $y \preceq_{n e} x$.

The following theorem was proved by de Mottoni and Schiaffino [19] for the Poincaré map of a periodic competitive Lotka-Volterra system of differential equations. Smith generalized the proof to competitive and cooperative maps [12].

Theorem 2 Let $\mathcal{S}$ be a nonempty subset of $\mathbb{R}^{2}$. If $T$ is a competitive map for which $\left(\mathrm{O}_{+}\right)$ holds, then, for all $x \in \mathcal{S},\left\{T^{n}(x)\right\}$ is eventually component-wise monotone. If the orbit of $x$ has compact closure, then it converges to a fixed point of T. If instead (O-) holds, then, for all $x \in \mathcal{S},\left\{T^{2 n}(x)\right\}$ is eventually component-wise monotone. If the orbit of $x$ has compact closure in $\mathcal{S}$, then its omega limit set is either a period-two orbit or a fixed point.

The following result is from [14], with the domain of the map specialized to be the cartesian product of intervals of real numbers. It gives a sufficient condition for conditions $\left(\mathrm{O}_{+}\right)$ and $(O-)$.

Theorem 3 Let $\mathcal{R} \subset \mathbb{R}^{2}$ be the cartesian product of two intervals in $\mathbb{R}$. Let $T: \mathcal{R} \rightarrow \mathcal{R}$ be a $C^{1}$ competitive map. If $T$ is injective and $\operatorname{det} J_{T}(x)>0$ for all $x \in \mathcal{R}$, then $T$ satisfies $(O+)$. If $T$ is injective and $\operatorname{det} J_{T}(x)<0$ for all $x \in \mathcal{R}$, then $T$ satisfies $(O-)$.

The following result is a direct consequence of the trichotomy theorem of Dancer and Hess, see [2] and [25], and is helpful for determining the basins of attraction of the equilibrium points.

Corollary 1 If the nonnegative cone of $\preceq$ is a generalized quadrant in $\mathbb{R}^{n}$, and if $T$ has no fixed points in $\llbracket u_{1}, u_{2} \rrbracket$ other than $u_{1}$ and $u_{2}$, then the interior of $\llbracket u_{1}, u_{2} \rrbracket$ is either a subset of the basin of attraction of $u_{1}$ or a subset of the basin of attraction of $u_{2}$.

The next result is a well-known global attractivity result which holds in partially ordered Banach spaces as well, see [25]. 
Theorem 4 Let $T$ be a monotone map on a closed and bounded rectangular region $\mathcal{R} \subset \mathbb{R}^{2}$. Suppose that $T$ has a unique fixed point $\overline{\mathbf{e}}$ in $\mathcal{R}$. Then $\overline{\mathbf{e}}$ is a global attractor of $T$ on $\mathcal{R}$.

The following theorems were proved by Kulenović and Merino [3] for competitive systems in the plane, when one of the eigenvalues of the linearized system at an equilibrium (hyperbolic or non-hyperbolic) is by an absolute value smaller than 1 , while the other has an arbitrary value. These results are useful for determining basins of attraction of fixed points of competitive maps.

Theorem 5 Let $T$ be a competitive map on a rectangular region $\mathcal{R} \subset \mathbb{R}^{2}$. Let $\overline{\mathrm{x}} \in \mathcal{R}$ be a fixed point of $T$ such that $\Delta:=\mathcal{R} \cap \operatorname{int}\left(\mathcal{Q}_{1}(\bar{x}) \cup \mathcal{Q}_{3}(\overline{\mathrm{x}})\right)$ is nonempty (i.e., $\overline{\mathrm{x}}$ is not the $N W$ or $S E$ vertex of $\mathcal{R})$, and $T$ is strongly competitive on $\Delta$. Suppose that the following statements are true.

(a) The map $T$ has a $C^{1}$ extension to a neighborhood of $\overline{\mathrm{x}}$.

(b) The Jacobian $J_{T}(\overline{\mathrm{x}})$ of $T$ at $\overline{\mathrm{x}}$ has real eigenvalues $\lambda$, $\mu$ such that $0<|\lambda|<\mu$, where $|\lambda|<1$, and the eigenspace $E^{\lambda}$ associated with $\lambda$ is not a coordinate axis.

Then there exists a curve $\mathcal{C} \subset \mathcal{R}$ through $\overline{\mathrm{x}}$ that is invariant and a subset of the basin of attraction of $\overline{\mathrm{x}}$ such that $\mathcal{C}$ is tangential to the eigenspace $E^{\lambda}$ at $\overline{\mathrm{x}}$, and $\mathcal{C}$ is the graph of a strictly increasing continuous function of the first coordinate on an interval. Any endpoints of $\mathcal{C}$ in the interior of $\mathcal{R}$ are either fixed points or minimal period-two points. In the latter case, the set of endpoints of $\mathcal{C}$ is a minimal period-two orbit of $T$.

The situation where the endpoints of $\mathcal{C}$ are boundary points of $\mathcal{R}$ is of interest. The following result gives a sufficient condition for this case.

Theorem 6 For the curve $\mathcal{C}$ of Theorem 5 to have endpoints in $\partial \mathcal{R}$, it is sufficient that at least one of the following conditions is satisfied.

(i) The map $T$ has no fixed points nor periodic points of minimal period two in $\Delta$.

(ii) The map $T$ has no fixed points in $\Delta, \operatorname{det} J_{T}(\overline{\mathrm{x}})>0$, and $T(\mathrm{x})=\overline{\mathrm{x}}$ has no solutions $\mathrm{x} \in \Delta$.

(iii) The map $T$ has no points of minimal period two in $\Delta$, $\operatorname{det} J_{T}(\overline{\mathrm{x}})<0$, and $T(\mathrm{x})=\overline{\mathrm{x}}$ has no solutions $\mathrm{x} \in \Delta$.

The next result is useful for determining basins of attraction of fixed points of competitive maps.

Theorem 7 (A) Assume the hypotheses of Theorem 5, and let $\mathcal{C}$ be the curve whose existence is guaranteed by Theorem 5. If the endpoints of $\mathcal{C}$ belong to $\partial \mathcal{R}$, then $\mathcal{C}$ separates $\mathcal{R}$ into two connected components, namely

$$
\mathcal{W}_{-}:=\left\{\mathrm{x} \in \mathcal{R} \backslash \mathcal{C}: \exists \mathrm{y} \in \mathcal{C} \text { with } \mathrm{x} \preceq_{\text {se }} \mathrm{y}\right\} \quad \text { and } \quad \mathcal{W}_{+}:=\left\{\mathrm{x} \in \mathcal{R} \backslash \mathcal{C}: \exists \mathrm{y} \in \mathcal{C} \text { with } \mathrm{y} \preceq_{s e} \mathrm{x}\right\}
$$

such that the following statements are true.

(i) $\mathcal{W}_{-}$is invariant, and $\operatorname{dist}\left(T^{n}(\mathrm{x}), \mathcal{Q}_{2}(\overline{\mathrm{x}})\right) \rightarrow 0$ as $n \rightarrow \infty$ for every $\mathrm{x} \in \mathcal{W}_{-}$.

(ii) $\mathcal{W}_{+}$is invariant, and $\operatorname{dist}\left(T^{n}(\mathrm{x}), \mathcal{Q}_{4}(\overline{\mathrm{x}})\right) \rightarrow 0$ as $n \rightarrow \infty$ for every $\mathrm{x} \in \mathcal{W}_{+}$. 
(B) If, in addition to the hypotheses of part (A), $\overline{\mathrm{x}}$ is an interior point of $\mathcal{R}$ and $T$ is $C^{2}$ and strongly competitive in a neighborhood of $\overline{\mathrm{x}}$, then $T$ has no periodic points in the boundary of $\mathcal{Q}_{1}(\overline{\mathrm{x}}) \cup \mathcal{Q}_{3}(\overline{\mathrm{x}})$ except for $\overline{\mathrm{x}}$, and the following statements are true.

(iii) For every $\mathrm{x} \in \mathcal{W}_{-}$, there exists $n_{0} \in \mathbb{N}$ such that $T^{n}(\mathrm{x}) \in \operatorname{int} \mathcal{Q}_{2}(\overline{\mathrm{x}})$ for $n \geq n_{0}$.

(iv) For every $\mathrm{x} \in \mathcal{W}_{+}$, there exists $n_{0} \in \mathbb{N}$ such that $T^{n}(\mathrm{x}) \in \operatorname{int} \mathcal{Q}_{4}(\overline{\mathrm{x}})$ for $n \geq n_{0}$.

If $T$ is a map on a set $\mathcal{R}$ and if $\bar{x}$ is a fixed point of $T$, the stable set $\mathcal{W}^{s}(\overline{\mathrm{x}})$ of $\overline{\mathrm{x}}$ is the set $\left\{x \in \mathcal{R}: T^{n}(\mathrm{x}) \rightarrow \overline{\mathrm{x}}\right\}$ and unstable set $\mathcal{W}^{u}(\overline{\mathrm{x}})$ of $\overline{\mathrm{x}}$ is the set

$$
\left\{\mathrm{x} \in \mathcal{R} \text { : there exists }\left\{\mathrm{x}_{n}\right\}_{n=-\infty}^{0} \subset \mathcal{R} \text { s.t. } T\left(\mathrm{x}_{n}\right)=\mathrm{x}_{n+1}, \mathrm{x}_{0}=\mathrm{x} \text {, and } \lim _{n \rightarrow-\infty} \mathrm{x}_{n}=\overline{\mathrm{x}}\right\} .
$$

When $T$ is non-invertible, the set $\mathcal{W}^{s}(\overline{\mathrm{x}})$ may not be connected and made up of infinitely many curves, or $\mathcal{W}^{u}(\overline{\mathrm{x}})$ may not be a manifold. The following result gives a description of the stable and unstable sets of a saddle point of a competitive map. If the map is a diffeomorphism on $\mathcal{R}$, the sets $\mathcal{W}^{s}(\overline{\mathrm{x}})$ and $\mathcal{W}^{u}(\overline{\mathrm{x}})$ are the stable and unstable manifolds of $\bar{x}$.

Theorem 8 In addition to the hypotheses of part (B) of Theorem 7, suppose that $\mu>1$ and that the eigenspace $E^{\mu}$ associated with $\mu$ is not a coordinate axis. If the curve $\mathcal{C}$ of Theorem 5 has endpoints in $\partial \mathcal{R}$, then $\mathcal{C}$ is the stable set $\mathcal{W}^{s}(\bar{x})$ of $\bar{x}$, and the unstable set $\mathcal{W}^{u}(\bar{x})$ of $\bar{x}$ is a curve in $\mathcal{R}$ that is tangential to $E^{\mu}$ at $\overline{\mathrm{x}}$ and such that it is the graph of a strictly decreasing function of the first coordinate on an interval. Any endpoints of $\mathcal{W}^{u}(\bar{x})$ in $\mathcal{R}$ are fixed points of $T$.

The following result gives information on local dynamics near a fixed point of a map when there exists a characteristic vector whose coordinates have negative product and such that the associated eigenvalue is hyperbolic. This is a well-known result, valid in a much more general setting: we include it here for completeness. A point $(x, y)$ is a subsolution if $T(x, y) \preceq_{s e}(x, y)$, and $(x, y)$ is a supersolution if $(x, y) \preceq_{s e} T(x, y)$. An order interval $\llbracket(a, b),(c, d) \rrbracket$ is the cartesian product of the two compact intervals $[a, c]$ and $[b, d]$.

Theorem 9 Let $T$ be a competitive map on a rectangular set $\mathcal{R} \subset \mathbb{R}^{2}$ with an isolated fixed point $\overline{\mathrm{x}} \in \mathcal{R}$ such that $\mathcal{R} \cap \operatorname{int}\left(\mathcal{Q}_{2}(\overline{\mathrm{x}}) \cup \mathcal{Q}_{4}(\overline{\mathrm{x}})\right) \neq \emptyset$. Suppose that $T$ has a $C^{1}$ extension to a neighborhood of $\overline{\mathrm{x}}$. Let $\mathrm{v}=\left(\mathrm{v}^{(1)}, \mathrm{v}^{(2)}\right) \in \mathbb{R}^{2}$ be an eigenvector of the Jacobian of $T$ at $\overline{\mathrm{x}}$, with associated eigenvalue $\mu \in \mathbb{R}$. If $\mathrm{v}^{(1)} \mathrm{v}^{(2)}<0$, then there exists an order interval $\mathcal{I}$ which is also a relative neighborhood of $\overline{\mathrm{x}}$ such that for every relative neighborhood $\mathcal{U} \subset \mathcal{I}$ of $\overline{\mathrm{x}}$ the following statements are true.

(i) If $\mu>1$, then $\mathcal{U} \cap$ int $\mathcal{Q}_{2}(\overline{\mathrm{x}})$ contains a subsolution and $\mathcal{U} \cap$ int $\mathcal{Q}_{4}(\overline{\mathrm{x}})$ contains $a$ supersolution. In this case, for every $\mathrm{x} \in \mathcal{I} \cap \operatorname{int}\left(\mathcal{Q}_{2}(\overline{\mathrm{x}}) \cup \mathcal{Q}_{4}(\overline{\mathrm{x}})\right)$, there exists $N$ such that $T^{n}(\mathrm{x}) \notin \mathcal{I}$ for $n \geq N$.

(ii) If $\mu<1$, then $\mathcal{U} \cap$ int $\mathcal{Q}_{2}(\overline{\mathrm{x}})$ contains a supersolution and $\mathcal{U} \cap$ int $\mathcal{Q}_{4}(\overline{\mathrm{x}})$ contains a subsolution. In this case $T^{n}(\mathrm{x}) \rightarrow \overline{\mathrm{x}}$ for every $\mathrm{x} \in \mathcal{I}$. 


\section{Some basic facts}

In this section we give some basic facts which will be used later. The map $T$ associated to system (1) is given by

$$
T(x, y)=(f(x, y), g(x, y))=\left(\frac{b_{1} x^{2}}{A_{1}+y^{2}}, \frac{a_{2}+c_{2} y^{2}}{x^{2}}\right) .
$$

Let

$$
\mathcal{R}=\mathbb{R}_{+}^{2} \backslash\{(0, y): y \geq 0\} .
$$

\subsection{Equilibrium points}

The equilibrium points $(\bar{x}, \bar{y})$ of system (1) satisfy the equations

$$
\frac{b_{1} \bar{x}^{2}}{\bar{y}^{2}+A_{1}}=\bar{x}, \quad \frac{c_{2} \bar{y}^{2}+a_{2}}{\bar{x}^{2}}=\bar{y} .
$$

By eliminating $\bar{x} \neq 0$ from (6), we get

$$
\bar{y}^{5}+2 A_{1} \bar{y}^{3}-b_{1}^{2} c_{2} \bar{y}^{2}+A_{1}^{2} \bar{y}-a_{2} b_{1}^{2}=0 .
$$

Similarly, we can eliminate variable $\bar{y}$ from system (6) to obtain

$$
b_{1} \bar{x}^{5}-A_{1} \bar{x}^{4}-b_{1}^{2} c_{2}^{2} \bar{x}^{2}-2 b_{1} c_{2}\left(a_{2}-A_{1} c_{2}\right) \bar{x}-\left(a_{2}-A_{1} c_{2}\right)^{2}=0 .
$$

Lemma 1 Let

$$
\begin{aligned}
\Delta_{1}= & -7,500 a_{2}^{3} A_{1} b_{1}^{4} c_{2}+a_{2}^{2}\left(5,550 A_{1}^{2} b_{1}^{4} c_{2}^{2}+256 A_{1}^{5}\right) \\
& -4 a_{2}\left(283 A_{1}^{3} b_{1}^{4} c_{2}^{3}+128 A_{1}^{6} c_{2}-27 b_{1}^{8} c_{2}^{5}\right)+3,125 a_{2}^{4} b_{1}^{4}-27 A_{1}^{4} b_{1}^{4} c_{2}^{4}+256 A_{1}^{7} c_{2}^{2}
\end{aligned}
$$

and

$$
\Delta_{2}=-460 a_{2} A_{1}^{2} c_{2}+250 a_{2}^{2} A_{1}+202 A_{1}^{3} c_{2}^{2}-27 b_{1}^{4} c_{2}^{4} .
$$

Then the following statements hold:

(a) Consider equation (7). Then all its real roots are positive numbers. Furthermore, equation (7) has one, two, or three real roots.

(b) If $\Delta_{1}>0$, then equation (7) has one real root and two pairs of distinct conjugate imaginary roots.

(c) If $\Delta_{1}<0$, then equation (7) has three distinct real roots and one pair of conjugate imaginary roots.

(d) If $\Delta_{1}=0$ and $\Delta_{2} \neq 0$, then equation (7) has one pair of conjugate imaginary roots and two real roots, one real root of multiplicity one and other one of multiplicity two.

(e) If $\Delta_{1}=0$ and $\Delta_{2}=0$, then equation (7) has one pair of conjugate imaginary roots and one real root of multiplicity three. 
Proof The proof of (a) follows from Descartes' rule of signs.

Let

$$
\tilde{f}(y)=y^{5}+2 A_{1} y^{3}-b_{1}^{2} c_{2} y^{2}+A_{1}^{2} y-a_{2} b_{1}^{2} .
$$

The following matrix, called the discrimination matrix of $\tilde{f}(y)$ and $\tilde{f}^{\prime}(y)$ in [26], is actually the Sylvester matrix of $\tilde{f}(y)$ and $\tilde{f}^{\prime}(y)$ with some permuted rows.

$$
\operatorname{Discr}(\tilde{f})=\left(\begin{array}{cccccccccc}
1 & 0 & 2 A_{1} & -b_{1}^{2} c_{2} & A_{1}^{2} & -a_{2} b_{1}^{2} & 0 & 0 & 0 & 0 \\
0 & 5 & 0 & 6 A_{1} & -2 b_{1}^{2} c_{2} & A_{1}^{2} & 0 & 0 & 0 & 0 \\
0 & 1 & 0 & 2 A_{1} & -b_{1}^{2} c_{2} & A_{1}^{2} & -a_{2} b_{1}^{2} & 0 & 0 & 0 \\
0 & 0 & 5 & 0 & 6 A_{1} & -2 b_{1}^{2} c_{2} & A_{1}^{2} & 0 & 0 & 0 \\
0 & 0 & 1 & 0 & 2 A_{1} & -b_{1}^{2} c_{2} & A_{1}^{2} & -a_{2} b_{1}^{2} & 0 & 0 \\
0 & 0 & 0 & 5 & 0 & 6 A_{1} & -2 b_{1}^{2} c_{2} & A_{1}^{2} & 0 & 0 \\
0 & 0 & 0 & 1 & 0 & 2 A_{1} & -b_{1}^{2} c_{2} & A_{1}^{2} & -a_{2} b_{1}^{2} & 0 \\
0 & 0 & 0 & 0 & 5 & 0 & 6 A_{1} & -2 b_{1}^{2} c_{2} & A_{1}^{2} & 0 \\
0 & 0 & 0 & 0 & 1 & 0 & 2 A_{1} & -b_{1}^{2} c_{2} & A_{1}^{2} & -a_{2} b_{1}^{2} \\
0 & 0 & 0 & 0 & 0 & 5 & 0 & 6 A_{1} & -2 b_{1}^{2} c_{2} & A_{1}^{2}
\end{array}\right) .
$$

Let $D_{k}$ denote the determinant of the submatrix of $\operatorname{Discr}(\tilde{f})$, formed by the first $2 k$ row and the first $2 k$ columns, for $k=1, \ldots, m$. So, by a straightforward calculation, one can see that

$$
\begin{aligned}
D_{1}= & 5 \\
D_{2}= & -20 A_{1} \\
D_{3}= & -16 A_{1}^{3}-45 b_{1}^{4} c_{2}^{2}, \\
D_{4}= & 2 b_{1}^{4}\left(-460 a_{2} A_{1}^{2} c_{2}+250 a_{2}^{2} A_{1}+202 A_{1}^{3} c_{2}^{2}-27 b_{1}^{4} c_{2}^{4}\right)=2 b_{1}^{4} \Delta_{2}, \\
D_{5}= & b_{1}^{4}\left(-7,500 a_{2}^{3} A_{1} b_{1}^{4} c_{2}+a_{2}^{2}\left(5,550 A_{1}^{2} b_{1}^{4} c_{2}^{2}+256 A_{1}^{5}\right)\right. \\
& -4 a_{2}\left(283 A_{1}^{3} b_{1}^{4} c_{2}^{3}+128 A_{1}^{6} c_{2}-27 b_{1}^{8} c_{2}^{5}\right) \\
& \left.+3,125 a_{2}^{4} b_{1}^{4}-27 A_{1}^{4} b_{1}^{4} c_{2}^{4}+256 A_{1}^{7} c_{2}^{2}\right)=b_{1}^{4} \Delta_{1} .
\end{aligned}
$$

Assume that $D_{5}>0$. The sign list of the sequence $\left\{D_{1}, D_{2}, D_{3}, D_{4}, D_{5}\right\}$ is given by

$$
\left[1,-1,-1, \operatorname{sign}\left(D_{4}\right), 1\right]
$$

from which it follows that the number of sign changes of the revised sign list of list (9) is two. Now, statement (b) follows in view of Theorem 1 [26]. Assume that $D_{5}<0$. If $D_{4} \geq 0$, then we obtain that $\tilde{f}(y)$ has three pairs of conjugate imaginary roots, which is a contradiction. Hence, $D_{4}<0$. The sign list of the sequence $\left\{D_{1}, D_{2}, D_{3}, D_{4}, D_{5}\right\}$ is given by

$$
[1,-1,-1,-1,-1]
$$

which implies that the number of sign changes of the revised sign list of (10) is one. Now, statement (c) follows in view of Theorem 1 [26]. Similarly, one can prove statements (d) and (e). 


\subsection{Injectivity, $\left(O_{+}\right)$and $\left(O_{-}\right)$}

Lemma 2 Assume that $(\bar{x}, \bar{y})$ is an equilibrium of the map $T$. Then the following hold:

(1) If $a_{2} \neq A_{1} c_{2}$, then $T$ is injective.

(2) If $a_{2}=A_{1} c_{2}$, then the curve

$$
b_{1} x^{2}=\bar{x}\left(A_{1}+y^{2}\right)
$$

is invariant under the map T. Furthermore, the following holds:

$$
T\left(x, \sqrt{\frac{b_{1} x^{2}-A_{1} \bar{x}}{\bar{x}}}\right)=(\bar{x}, \bar{y}) \quad \text { for } x \geq \sqrt{\frac{A_{1} \bar{x}}{b_{1}}} .
$$

(3) If $A_{1} c_{2}>a_{2}$, then $T$ satisfies $(O+)$, in which case $\left\{T^{n}\left(x_{0}, y_{0}\right)\right\}$ is asymptotic to either $(0, \infty)$ or $(\infty, 0)$, or to an equilibrium point, for all $\left(x_{0}, y_{0}\right) \in \mathcal{R}$.

(4) If $A_{1} c_{2}<a_{2}$, then $T$ satisfies $(O-)$, in which case $\left\{T^{n}\left(x_{0}, y_{0}\right)\right\}$ is asymptotic to either $(0, \infty)$ or $(\infty, 0)$, or to a period-two point, for all $\left(x_{0}, y_{0}\right) \in \mathcal{R}$.

Proof (1) Assume that $T\left(x_{1}, y_{1}\right)=T\left(x_{2}, y_{2}\right)$. Then we have

$$
\left(\frac{A_{1} b_{1} x_{1}^{2}-A_{1} b_{1} x_{2}^{2}+b_{1} x_{1}^{2} y_{2}^{2}-b_{1} x_{2}^{2} y_{1}^{2}}{\left(A_{1}+y_{1}^{2}\right)\left(A_{1}+y_{2}^{2}\right)}, \frac{-a_{2} x_{1}^{2}+a_{2} x_{2}^{2}-c_{2} x_{1}^{2} y_{2}^{2}+c_{2} x_{2}^{2} y_{1}^{2}}{x_{1}^{2} x_{2}^{2}}\right)=(0,0) .
$$

Equation (11) is equivalent to

$$
\begin{aligned}
& x_{1}^{2}\left(A_{1} b_{1}+b_{1} y_{2}^{2}\right)-A_{1} b_{1} x_{2}^{2}-b_{1} x_{2}^{2} y_{1}^{2}=0, \\
& x_{1}^{2}\left(-a_{2}-c_{2} y_{2}^{2}\right)+a_{2} x_{2}^{2}+c_{2} x_{2}^{2} y_{1}^{2}=0 .
\end{aligned}
$$

Equation (12) implies

$$
x_{1}^{2}=\frac{x_{2}^{2}\left(A_{1}+y_{1}^{2}\right)}{A_{1}+y_{2}^{2}} .
$$

By substituting this into equation (13), we obtain

$$
\frac{x_{2}^{2}\left(y_{1}^{2}-y_{2}^{2}\right)\left(a_{2}-A_{1} c_{2}\right)}{A_{1}+y_{2}^{2}}=0 \text {, }
$$

from which it follows that $y_{1}=y_{2}$ since $a_{2} \neq A_{1} c_{2}$. From (14) we have $x_{1}=x_{2}$, which completes the proof of statement (a).

(2) One can see that

$$
T\left(x, \sqrt{\frac{b_{1} x^{2}-A_{1} \bar{x}}{\bar{x}}}\right)-(\bar{x}, \bar{y})=\left(0, \frac{b_{1} c_{2}-\bar{x} \bar{y}}{\bar{x}}\right) .
$$

Since $a_{2}=A_{1} c_{2}$, equations (7) and ( 8 ) become

$$
c_{2}\left(\bar{y}^{2}+A_{1}\right)\left(A_{1} \bar{y}+\bar{y}^{3}-b_{1}^{2} c_{2}\right)=0
$$


and

$$
c_{2}-\bar{x}^{2}\left(-A_{1} \bar{x}^{2}+b_{1} \bar{x}^{3}-b_{1}^{2} c_{2}^{2}\right)=0 .
$$

From (15) we have $A_{1}=\frac{b_{1}^{2} c_{2}-\bar{y}^{3}}{\bar{y}}$. By substituting this into (16) we get

$$
\frac{\left(\bar{x} \bar{y}-b_{1} c_{2}\right)\left(b_{1}\left(c_{2} \bar{y}+\bar{x}^{2}\right)+\bar{x} \bar{y}^{2}\right)}{\bar{y}}=0,
$$

which implies $\bar{x} \bar{y}-b_{1} c_{2}=0$, from which the proof follows.

(3) The Jacobian matrix of the map $T$ has the form

$$
J_{T}=\left(\begin{array}{cc}
\frac{2 x b_{1}}{y^{2}+A_{1}} & -\frac{2 x^{2} y b_{1}}{\left(y^{2}+A_{1}\right)^{2}} \\
-\frac{2\left(c_{2} y^{2}+a_{2}\right)}{x^{3}} & \frac{2 y c_{2}}{x^{2}}
\end{array}\right) .
$$

The determinant of (17) at any point is equal to

$$
J_{T}(x, y)=\frac{4 b_{1} x^{2} y\left(A_{1} c_{2}-a_{2}\right)}{x^{3}\left(y^{2}+A_{1}\right)^{2}} .
$$

The proof of (3) and (4) follows from Theorem 3.

\section{Linearized stability analysis}

The determinant of (17) at the equilibrium point is given by

$$
\operatorname{det} J_{T}(\bar{x}, \bar{y})=\frac{4 b_{1} \bar{y}\left(A_{1} c_{2}-a_{2}\right)}{\bar{x}\left(\bar{y}^{2}+A_{1}\right)^{2}} .
$$

The trace of (17) at the equilibrium point is given by

$$
\operatorname{tr} J_{T}(\bar{x}, \bar{y})=\frac{2 c_{2} \bar{y}}{\bar{x}^{2}}+2
$$

The characteristic equation has the form

$$
\lambda^{2}-\lambda\left(\frac{2 c_{2} \bar{y}}{\bar{x}^{2}}+2\right)+\frac{4 b_{1} \bar{y}\left(A_{1} c_{2}-a_{2}\right)}{\bar{x}\left(\bar{y}^{2}+A_{1}\right)^{2}}=0 .
$$

Equilibrium curves $\mathcal{C}_{f}=\{(x, y) \in \mathcal{R}: f(x, y)=x\}$ and $\mathcal{C}_{g}=\{(x, y) \in \mathcal{R}: g(x, y)=y\}$ can be given explicitly as functions of $y$ :

$$
\begin{aligned}
& \mathcal{C}_{f}: x_{f}(y)=\frac{A_{1}+y^{2}}{b_{1}}, \\
& \mathcal{C}_{g}: \begin{cases}x_{g^{+}}(y)=+\sqrt{\frac{a_{2}+c_{2} y^{2}}{y}}, & y>0, \\
x_{g^{-}}(y)=-\sqrt{\frac{a_{2}+c_{2} y^{2}}{y}}, & y>0 .\end{cases}
\end{aligned}
$$

Note that $x_{g^{-}}(x)$ is always negative. Let $x_{g}(y)$ denote $x_{g^{+}}(y)$. We consider only $x_{f}(y)$ and $x_{g}(y)$. Let

$$
\tilde{x}(y)=x_{f}(y)-x_{g}(y)
$$


Lemma 3 Let $T=(f, g)$ be the map defined by (5). Then $f_{x}^{\prime}(x, y)>1$, and the following is true:

$$
\operatorname{sign}(\tilde{x}(y))=\operatorname{sign}\left(y^{5}+2 A_{1} y^{3}-b_{1}^{2} c_{2} y^{2}+A_{1}^{2} y-a_{2} b_{1}^{2}\right) .
$$

Proof The first derivative of $x_{f}(y)$ is given by

$$
x_{f}^{\prime}(y)=\frac{f_{y}^{\prime}(x, y)}{1-f_{x}^{\prime}(x, y)}=\frac{2 y}{b_{1}}>0 .
$$

Since $f_{y}^{\prime}(x, y)<0$, we get $f_{x}^{\prime}(x, y)>1$. Further,

$$
\tilde{x}(y)=x_{f}(y)-x_{g}(y)=\frac{A_{1}+y^{2}}{b_{1}}-\sqrt{\frac{a_{2}+c_{2} y^{2}}{y}}=\frac{\sqrt{y}\left(A_{1}+y^{2}\right)-b_{1} \sqrt{a_{2}+c_{2} y^{2}}}{b_{1} \sqrt{y}} .
$$

Now, the proof follows from

$$
\left(\sqrt{y}\left(A_{1}+y^{2}\right)\right)^{2}-\left(b_{1} \sqrt{a_{2}+c_{2} y^{2}}\right)^{2}=y^{5}+2 A_{1} y^{3}-b_{1}^{2} c_{2} y^{2}+A_{1}^{2} y-a_{2} b_{1}^{2} .
$$

Lemma 4 Let $T$ be the map defined by (5), and let

$$
J_{T}(\bar{x}, \bar{y})=\left(\begin{array}{ll}
a & b \\
c & d
\end{array}\right)
$$

be the Jacobian matrix of $T$ at a fixed point $(\bar{x}, \bar{y})$. Then the Jacobian matrix (19) has real and distinct eigenvalues $\lambda_{1}$ and $\lambda_{2}$ such that $\left|\lambda_{1}\right|<\lambda_{2}$ and $\lambda_{2}>1$. Furthermore, the following holds:

$$
\operatorname{sign}\left(\tilde{x}^{\prime}(\bar{y})\right)=\operatorname{sign}\left(1-\lambda_{1}\right) .
$$

Proof Implicit differentiation of the equations defining $C_{f}$ and $C_{g}$ at $(\bar{x}, \bar{y})$ gives

$$
x_{f}^{\prime}(\bar{y})=\frac{f_{y}^{\prime}(\bar{x}, \bar{y})}{1-f_{x}^{\prime}(\bar{x}, \bar{y})}, \quad x_{g}^{\prime}(\bar{y})=\frac{1-g_{y}^{\prime}(\bar{x}, \bar{y})}{g_{x}^{\prime}(\bar{x}, \bar{y})} .
$$

The characteristic equation associated with the Jacobian matrix of $T$ at $(\bar{x}, \bar{y})$ is given by

$$
\begin{aligned}
p(\lambda) & =\lambda^{2}-\left[f_{x}^{\prime}(\bar{x}, \bar{y})+g_{y}^{\prime}(\bar{x}, \bar{y})\right] \lambda+\left[f_{x}^{\prime}(\bar{x}, \bar{y}) g_{y}^{\prime}(\bar{x}, \bar{y})-f_{y}^{\prime}(\bar{x}, \bar{y}) g_{x}^{\prime}(\bar{x}, \bar{y})\right] \\
& =\lambda^{2}-(a+d) \lambda+(a d-b c) .
\end{aligned}
$$

Since the map $T$ is competitive, then the eigenvalues of the Jacobian matrix of the map $T$ at the equilibrium $(\bar{x}, \bar{y})$ are real and distinct and $\left|\lambda_{1}\right|<\lambda_{2}$. By (20), we have

$$
\begin{aligned}
\tilde{x}^{\prime}(\bar{y}) & =x_{f}^{\prime}(\bar{y})-x_{g}^{\prime}(\bar{y})=\frac{f_{y}^{\prime}(\bar{x}, \bar{y})}{1-f_{x}^{\prime}(\bar{x}, \bar{y})}-\frac{1-g_{y}^{\prime}(\bar{x}, \bar{y})}{g_{x}^{\prime}(\bar{x}, \bar{y})} \\
& =\frac{b}{1-a}-\frac{1-d}{c}=\frac{-1+(a+d)-(a d-b c)}{c(1-a)} \\
& =\frac{-p(1)}{c(1-a)}=\frac{\left(1-\lambda_{1}\right)\left(1-\lambda_{2}\right)}{c(a-1)} .
\end{aligned}
$$


From $\operatorname{tr} J_{T}(\bar{x}, \bar{y})=\lambda_{1}+\lambda_{2}>2$ we get $\lambda_{2}>1$. The map $T$ is competitive, which implies $c=g_{x}^{\prime}(\bar{x}, \bar{y})<0$. In view of Lemma 3 , we get $a=f_{x}^{\prime}(\bar{x}, \bar{y})>1$, from which it follows that $\operatorname{sign}\left(\tilde{x}^{\prime}(\bar{y})\right)=\operatorname{sign}\left(1-\lambda_{1}\right)$.

Theorem 10 Assume that $\Delta_{1}>0$ and $\lambda_{1}$ and $\lambda_{2}$ are eigenvalues of $J_{T}(\bar{x}, \bar{y})$. Then there exists the unique equilibrium point $E=(\bar{x}, \bar{y})$ and the following hold:

(a) If $a_{2}<A_{1} c_{2}$, then $E$ is a saddle point and $0<\lambda_{1}<1, \lambda_{2}>1$.

(b) Assume that $a_{2}>A_{1} c_{2}$. Let

$$
\Gamma(\bar{y})=5 c_{2} \bar{y}^{4}+\bar{y}^{2}\left(9 A_{1} c_{2}-a_{2}\right)+3 a_{2} A_{1} .
$$

(b1) If $\Gamma(\bar{y})>0$, then $E$ is a saddle point. Furthermore, the following hold:

$$
-1<\lambda_{1}<0, \lambda_{2}>1 \text {. }
$$

(b2) If $\Gamma(\bar{y})<0$, then $E$ is a repeller. Furthermore, the following hold: $\lambda_{1}<-1, \lambda_{2}>1$;

$$
\left|\lambda_{1}\right|<\lambda_{2} \text {. }
$$

(b3) If $\Gamma(\bar{y})=0$, then $E$ is a non-hyperbolic equilibrium point. Furthermore, the following hold: $\lambda_{1}=-1, \lambda_{2}>1$.

Proof In view of (7) and Lemma 1, we have that the function

$$
\tilde{f}(y)=y^{5}+2 A_{1} y^{3}-b_{1}^{2} c_{2} y^{2}+A_{1}^{2} y-a_{2} b_{1}^{2}
$$

has one zero $\bar{y}$ of multiplicity one. In view of Lemma 1 , the map $T$ has a unique equilibrium point. Since $\tilde{f}(0)=-a_{2} b_{1}^{2}<0$ and $\lim _{y \rightarrow+\infty} \tilde{f}(y)=+\infty$, we have $\tilde{f}(y)<0$ for $y<\bar{y}$ and $\tilde{f}(y)>$ 0 for $y>\bar{y}$. By Lemmas 6 and 7 from [27], the equilibrium curves $\mathcal{C}_{f}$ and $\mathcal{C}_{g}$ intersect transversally at $(\bar{x}, \bar{y})$, i.e., $\tilde{x}^{\prime}(\bar{y}) \neq 0$. In view of Lemma 3 and by the continuity of function $\tilde{x}(y)$, there exists a neighborhood $U_{\bar{y}}$ of $\bar{y}$ such that $\tilde{x}^{\prime}(y)>0$ for $y \in U_{\bar{y}}$, which implies

$$
\tilde{x}^{\prime}(\bar{y})=x_{f}^{\prime}(\bar{y})-x_{g}^{\prime}(\bar{y})>0 .
$$

From (21) and Lemma 4 we obtain $\lambda_{1}<1$ and $\lambda_{2}>1$.

If $a_{2}<A_{1} c_{2}$, then $\operatorname{det} J_{T}(\bar{x}, \bar{y})=\lambda_{1} \lambda_{2}>0$, which implies that $\lambda_{1} \in(0,1)$.

Now, assume that $a_{2}>A_{1} c_{2}$. By using

$$
b_{1}=\frac{\bar{y}^{2}+A_{1}}{\bar{x}}, \quad \bar{x}=\sqrt{\frac{c_{2} \bar{y}^{2}+a_{2}}{\bar{y}}},
$$

one can see that

$$
\begin{aligned}
p(-1) & =1+\operatorname{det} J_{T}(\bar{x}, \bar{y})+\operatorname{tr} J_{T}(\bar{x}, \bar{y})=\frac{5 c_{2} \bar{y}^{4}+\bar{y}^{2}\left(9 A_{1} c_{2}-a_{2}\right)+3 a_{2} A_{1}}{\left(\bar{y}^{2}+A_{1}\right)\left(c_{2} \bar{y}^{2}+a_{2}\right)} \\
& =\frac{\Gamma(\bar{y})}{\left(\bar{y}^{2}+A_{1}\right)\left(c_{2} \bar{y}^{2}+a_{2}\right)}
\end{aligned}
$$

and

$$
\operatorname{det} J_{T}(\bar{x}, \bar{y})=\lambda_{1} \lambda_{2}=\frac{4 b_{1} \bar{y}\left(A_{1} c_{2}-a_{2}\right)}{\bar{x}\left(\bar{y}^{2}+A_{1}\right)^{2}}
$$


where $p(\lambda)=\left(\lambda-\lambda_{1}\right)\left(\lambda-\lambda_{2}\right)$. In view of $(18)$ and $p(-1)=\left(\lambda_{1}+1\right)\left(\lambda_{2}+1\right)$, we obtain statement (b) of the theorem.

Lemma 5 Suppose that all the assumptions of Theorem 10 are satisfied. Let

$$
y_{ \pm}=\sqrt{\frac{a_{2}-9 A_{1} c_{2} \pm \sqrt{-78 a_{2} A_{1} c_{2}+a_{2}^{2}+81 A_{1}^{2} c_{2}^{2}}}{10 c_{2}}} .
$$

Then the following statements are true.

(a) $\Gamma(\bar{y})>0$ if and only if one of the following inequalities holds:

$$
\begin{aligned}
& 9 A_{1} c_{2}-a_{2} \geq 0 \text {, } \\
& 9 A_{1} c_{2}-a_{2}<0 \text { and }-78 a_{2} A_{1} c_{2}+a_{2}^{2}+81 A_{1}^{2} c_{2}^{2}<0, \\
& 9 A_{1} c_{2}-a_{2}<0 \text { and }-78 a_{2} A_{1} c_{2}+a_{2}^{2}+81 A_{1}^{2} c_{2}^{2} \geq 0 \text { and } \\
& \left(\tilde{f}\left(y_{-}\right)>0 \text { or } \tilde{f}\left(y_{+}\right)<0\right) ;
\end{aligned}
$$

(b) $\Gamma(\bar{y})<0$ if and only if the following hold:

$$
9 A_{1} c_{2}-a_{2}<0 \text { and }-78 a_{2} A_{1} c_{2}+a_{2}^{2}+81 A_{1}^{2} c_{2}^{2} \geq 0 \text { and }\left(\tilde{f}\left(y_{-}\right)<0 \text { and } \tilde{f}\left(y_{+}\right)>0\right) \text {; }
$$

(c) $\Gamma(\bar{y})=0$ if and only if

$$
9 A_{1} c_{2}-a_{2}<0 \text { and }-78 a_{2} A_{1} c_{2}+a_{2}^{2}+81 A_{1}^{2} c_{2}^{2} \geq 0 \quad \text { and } \quad\left(\tilde{f}\left(y_{-}\right)=0 \text { or } \tilde{f}\left(y_{+}\right)=0\right) \text {. }
$$

Proof The function $\tilde{f}(y)$ has one simple zero $\bar{y}$, which implies $\tilde{f}(y)<0$ for $0 \leq y<\bar{y}$ and $\tilde{f}(y)>0$ for $y>\bar{y}$. Then

$$
\bar{y}>\alpha \text { if and only if } \tilde{f}(\alpha)<0 \text {, }
$$

while

$$
\bar{y}<\beta \quad \text { if and only if } \tilde{f}(\beta)>0
$$

for some $\alpha, \beta \in[0, \infty)$. Now the proof follows from the fact that $\tilde{f}(y)=0$ has real roots

$$
\begin{aligned}
& \left\{\sqrt{\frac{a_{2}-9 A_{1} c_{2} \pm \sqrt{-78 a_{2} A_{1} c_{2}+a_{2}^{2}+81 A_{1}^{2} c_{2}^{2}}}{10 c_{2}}},\right. \\
& \left.-\sqrt{\frac{a_{2}-9 A_{1} c_{2} \pm \sqrt{-78 a_{2} A_{1} c_{2}+a_{2}^{2}+81 A_{1}^{2} c_{2}^{2}}}{10 c_{2}}}\right\}
\end{aligned}
$$

if and only if

$$
9 A_{1} c_{2}-a_{2}<0 \text { and }-78 a_{2} A_{1} c_{2}+a_{2}^{2}+81 A_{1}^{2} c_{2}^{2} \geq 0
$$


Theorem 11 Assume that $\Delta_{1}<0$. Then there exist three distinct equilibrium points in the positive quadrant: $E_{1}=\left(\bar{x}_{1}, \bar{y}_{1}\right), E_{2}=\left(\bar{x}_{2}, \bar{y}_{2}\right)$ and $E_{3}=\left(\bar{x}_{3}, \bar{y}_{3}\right)$ such that $E_{1} \ll_{n e} E_{2} \ll_{n e} E_{3}$ and the following hold:

(a) $E_{1}$ and $E_{3}$ are saddle points. If $\lambda_{1}^{(i)}$ and $\lambda_{2}^{(i)}$ are the eigenvalues of $J_{T}\left(E_{i}\right), i=1,3$, then $0<\lambda_{1}^{(i)}<1, \lambda_{2}^{(i)}>1$.

(b) The equilibrium point $E_{2}$ is a repeller. If $\lambda_{1}^{(2)}$ and $\lambda_{2}^{(2)}$ are the eigenvalues of $J_{T}\left(E_{2}\right)$, then $1<\lambda_{1}^{(2)}<\lambda_{2}^{(2)}$.

Proof In view of Lemma 1, equation (7) has three positive roots of multiplicity one. Since

$$
\bar{x}=\frac{A_{1}+\bar{y}^{2}}{b_{1}}>0,
$$

then by (a) of Lemma 1 we obtain that the map $T$ has three equilibrium points that we denote by $E_{1}, E_{2}$ and $E_{3}$. Given points lie on the increasing curve

$$
x_{f}(y)=\frac{A_{1}+y^{2}}{b_{1}},
$$

which implies that the points are in the north-east ordering. Descartes' rule of signs and (8) imply that $\operatorname{det} J_{T}(x, y)>0$ when $a_{2}<A_{1} c_{2}$. In view of (7) and Lemma 1 , we have that the polynomial

$$
\tilde{f}(y)=y^{5}+2 A_{1} y^{3}-b_{1}^{2} c_{2} y^{2}+A_{1}^{2} y-a_{2} b_{1}^{2}
$$

has three zeros $\bar{y}_{i}, i=1,2,3$, of multiplicity one. Since $\tilde{f}(0)=-a_{2} b_{1}^{2}<0$ and $\lim _{y \rightarrow+\infty} \tilde{f}(y)=$ $+\infty$, we have $\tilde{f}(y)<0$ for $y \in\left(0, \bar{y}_{1}\right) \cup\left(\bar{y}_{2}, \bar{y}_{3}\right)$ and $\tilde{f}(y)>0$ for $y \in\left(\bar{y}_{1}, \bar{y}_{2}\right) \cup\left(\bar{y}_{3},+\infty\right)$.

By Lemmas 6 and 7 from [27], the equilibrium curves $\mathcal{C}_{f}$ and $\mathcal{C}_{g}$ intersect transversally at $E_{1}, E_{2}$ and $E_{3}$, i.e., $\tilde{x}^{\prime}\left(\bar{y}_{i}\right) \neq 0, i=1,2,3$. By this and Lemma 3 and by the continuity of function $\tilde{x}(y)$, there exists a neighborhood $U_{\bar{y}_{i}}^{(i)}$ of $\bar{y}_{i}$ such that $\tilde{x}^{\prime}(y)>0$ for $y \in U_{\bar{y}_{i}}^{(i)}$ for $i=1,3$ and $\tilde{x}^{\prime}(y)<0$ for $y \in U_{\bar{y}_{i}}^{(2)}$. Using this we get

$$
\tilde{x}^{\prime}\left(\bar{y}_{i}\right)>0 \quad \text { for } i=1,3 \quad \text { and } \quad \tilde{x}^{\prime}\left(\bar{y}_{i}\right)<0 \quad \text { for } i=2 \text {. }
$$

Let

$$
J_{T}\left(E_{i}\right)=\left(\begin{array}{ll}
a_{i} & b_{i} \\
c_{i} & d_{i}
\end{array}\right), \quad i=1,2,3 .
$$

In view of (18), we have $\operatorname{det} J_{T}\left(E_{i}\right)=\lambda_{1}^{(i)} \lambda_{2}^{(i)}>0, i=1,2,3$. By Lemma 4 we obtain $0<\lambda_{1}^{(i)}<1$ and $\lambda_{2}^{(i)}>1$ for $i=1,3$. Since $\tilde{x}^{\prime}\left(\bar{y}_{2}\right)<0$, by Lemma 4 we have $1<\lambda_{1}^{(2)}<\lambda_{2}^{(2)}$. This completes the proof.

Theorem 12 Assume that $\Delta_{1}=0$ and $\Delta_{2} \neq 0$ Then there exist two distinct equilibrium points in the positive quadrant $E_{1}=\left(\bar{x}_{1}, \bar{y}_{1}\right)$ and $E_{3}=\left(\bar{x}_{3}, \bar{y}_{3}\right)$ such that $E_{1} \ll_{n e} E_{3}$. Let $\lambda_{1}^{(i)}$ and $\lambda_{2}^{(i)}$ be the eigenvalues of $J_{T}\left(E_{i}\right), i=1,3$. Then the following hold:

(a) Exactly one of the roots $\bar{y}_{1}$ or $\bar{y}_{3}$ of (7) has multiplicity two. 
(b) If $\bar{y}_{1}$ is a root of (7) of multiplicity two, then the equilibrium point $E_{1}$ is non-hyperbolic and $E_{3}$ is a saddle point. Furthermore, $\lambda_{1}^{(1)}=1, \lambda_{2}^{(1)}>1$ and $0<\lambda_{1}^{(3)}<1, \lambda_{2}^{(3)}>1$.

(c) If $\bar{y}_{3}$ is a root of (7) of multiplicity two, then the equilibrium point $E_{3}$ is non-hyperbolic and $E_{1}$ is a saddle point. Furthermore, $\lambda_{1}^{(3)}=1, \lambda_{2}^{(3)}>1$ and $0<\lambda_{1}^{(1)}<1, \lambda_{2}^{(1)}>1$.

Proof In view of Lemma 1, equation (7) has two positive zeros, one of multiplicity one and another one of multiplicity two, which implies statement (a). Since $\bar{x}=\left(A_{1}+\bar{y}^{2}\right) / b_{1}>0$, we obtain that the map $T$ has two equilibrium points that we denote by $E_{1}$ and $E_{3}$. Descartes' rule of signs and (8) imply that $a_{2}<A_{1} c_{2} \Rightarrow \operatorname{det} J_{T}(x, y)>0$. Now, we prove statement (b). Similarly as in the proof of Theorem 11, one can see that $E_{3}$ is a saddle point. In view of Lemmas 6 and 7, from [27] we have that $\tilde{x}^{\prime}\left(\bar{y}_{1}\right)=0$, since $\bar{y}_{1}$ is the root of (7) of multiplicity two. By Lemma 4 we obtain $\lambda_{1}^{(1)}=1, \lambda_{2}^{(1)}>1$. The proof of statement (c) is similar and we will skip it.

Theorem 13 Assume that $\Delta_{1}=0$ and $\Delta_{2}=0$. Then there exists one equilibrium point in the positive quadrant $E_{1}=\left(\bar{x}_{1}, \bar{y}_{1}\right)$ which is non-hyperbolic. If $\lambda_{1}^{(1)}$ and $\lambda_{2}^{(1)}$ are eigenvalues of $J_{T}\left(E_{1}\right)$, then $\lambda_{1}^{(1)}=1, \lambda_{2}^{(1)}>1$.

Proof In view of Lemma $1, \bar{y}_{1}$ is zero of (7) of multiplicity three. In view of Lemmas 6 and 7 , from [27] we have that $\tilde{x}^{\prime}\left(\bar{y}_{1}\right)=0$. The rest of the proof is similar to that in Theorem 12 and we skip it.

\subsection{Period-two solution}

Let

$$
T^{2}(x, y)=T(T(x, y))=(F(x, y), G(x, y)),
$$

where

$$
F(x, y)=\frac{b_{1}^{3} x^{8}}{\left(A_{1}+y^{2}\right)^{2}\left(2 a_{2} c_{2} y^{2}+a_{2}^{2}+A_{1} x^{4}+c_{2}^{2} y^{4}\right)}
$$

and

$$
G(x, y)=\frac{\left(A_{1}+y^{2}\right)^{2}\left(2 a_{2} c_{2}^{2} y^{2}+a_{2}^{2} c_{2}+a_{2} x^{4}+c_{2}^{3} y^{4}\right)}{b_{1}^{2} x^{8}} .
$$

Period-two solution $\{(\Phi, \Psi), T(\Phi, \Psi)\}$ satisfies the system

$$
F(\Phi, \Psi)=\Phi, \quad G(\Phi, \Psi)=\Psi
$$

which is equivalent to

$$
\begin{aligned}
& b_{1}^{3} \Phi^{7}-A_{1} \Phi^{4}\left(A_{1}+\Psi^{2}\right)^{2}-\left(A_{1}+\Psi^{2}\right)^{2}\left(a_{2}+c_{2} \Psi^{2}\right)^{2}=0, \\
& b_{1}^{2} \Psi \Phi^{8}-a_{2} \Phi^{4}\left(A_{1}+\Psi^{2}\right)^{2}-c_{2}\left(A_{1}+\Psi^{2}\right)^{2}\left(a_{2}+c_{2} \Psi^{2}\right)^{2}=0 .
\end{aligned}
$$


The Jacobian matrix of the map $T^{2}$ at $(x, y)$ has the form

$$
J_{T^{2}}(x, y)=\left(\begin{array}{cc}
\frac{4 x^{7} b_{1}^{3}\left(A_{1} x^{4}+2\left(c_{2} y^{2}+a_{2}\right)^{2}\right)}{\left(y^{2}+A_{1}\right)^{2}\left(A_{1} x^{4}+\left(c_{2} y^{2}+a_{2}\right)^{2}\right)^{2}} & -\frac{4 x^{8} y b_{1}^{3}\left(A_{1} x^{4}+\left(c_{2} y^{2}+a_{2}\right)\left(a_{2}+\left(2 y^{2}+A_{1}\right) c_{2}\right)\right)}{\left(y^{2}+A_{1}\right)^{3}\left(A_{1} x^{4}+\left(c_{2} y^{2}+a_{2}\right)^{2}\right)^{2}} \\
-\frac{4\left(y^{2}+A_{1}\right)^{2}\left(2 c_{2}^{3} y^{4}+2 a_{2}^{2} c_{2}+a_{2}\left(x^{4}+4 y^{2} c_{2}^{2}\right)\right)}{x^{3} b_{1}^{2}} & \frac{4 y\left(y^{2}+A_{1}\right)\left(y^{2}\left(2 y^{2}+A_{1}\right) c_{2}^{3}+a_{2}^{2} c_{2}+a_{2}\left(x^{4}+\left(3 y^{2}+A_{1}\right) c_{2}^{2}\right)\right)}{x^{8} b_{1}^{2}}
\end{array}\right) .
$$

The determinant of $(23)$ at $(x, y)$ is given by

$$
\operatorname{det} J_{T^{2}}(x, y)=\frac{16 b_{1} x^{3} y\left(a_{2}-A_{1} c_{2}\right)^{2}\left(a_{2}+c_{2} y^{2}\right)}{\left(A_{1}+y^{2}\right)\left(\left(a_{2}+c_{2} y^{2}\right)^{2}+A_{1} x^{4}\right)^{2}}
$$

The trace of $(23)$ at $(x, y)$ is given by

$$
\begin{aligned}
\operatorname{tr} J_{T^{2}}(x, y)= & \frac{4\left(\frac{b_{1}^{5} x^{15}\left(2\left(a_{2}+c_{2} y^{2}\right)^{2}+A_{1} x^{4}\right)}{\left(\left(a_{2}+c_{2} y^{2}\right)^{2}+A_{1} x^{4}\right)^{2}}+a_{2} y\left(A_{1}+y^{2}\right)^{3}\left(c_{2}^{2}\left(A_{1}+3 y^{2}\right)+x^{4}\right)\right)}{b_{1}^{2} x^{8}\left(A_{1}+y^{2}\right)^{2}} \\
& +\frac{4\left(a_{2}^{2} c_{2} y\left(A_{1}+y^{2}\right)^{3}+c_{2}^{3} y^{3}\left(A_{1}+2 y^{2}\right)\left(A_{1}+y^{2}\right)^{3}\right)}{b_{1}^{2} x^{8}\left(A_{1}+y^{2}\right)^{2}} .
\end{aligned}
$$

Lemma 6 Let $\mathcal{C}_{F}:=\{(x, y): F(x, y)=x\}$ and $\mathcal{C}_{G}:=\{(x, y): G(x, y)=y\}$ be the period-two curves, that is, the curves the intersection of which is a period-two solution. Then, for all $y>0$, there exist exactly one $x_{F}(y)>0$ and exactly one $x_{G}(y)>0$ such that $F\left(x_{F}(y), y\right)=x$ and $G\left(x_{G}(y), y\right)=y$. Furthermore, $x_{F}(y)$ and $x_{G}(y)$ are continuous functions and $x_{F}^{\prime}(y)>0$.

Proof Since $F(x, y)=x$ and $G(x, y)=y$ if and only if

$$
\begin{aligned}
& b_{1}^{3} x^{7}-A_{1} x^{4}\left(A_{1}+y^{2}\right)^{2}-\left(A_{1}+y^{2}\right)^{2}\left(a_{2}+c_{2} y^{2}\right)^{2}=0, \\
& b_{1}^{2} y x^{8}-a_{2} x^{4}\left(A_{1}+y^{2}\right)^{2}-c_{2}\left(A_{1}+y^{2}\right)^{2}\left(a_{2}+c_{2} y^{2}\right)^{2}=0,
\end{aligned}
$$

respectively, in view of Descartes' rule of signs, we have that for all $y>0$ there exist exactly one $x_{F}(y)>0$ and exactly one $x_{G}(y)>0$ such that $F\left(x_{F}(y), y\right)=x$ and $G\left(x_{G}(y), y\right)=y$. Taking derivatives of $F(x, y)=x$ with respect to $y$, we get

$$
x_{F}^{\prime}(y)=\frac{F_{y}^{\prime}(x, y)}{1-F_{x}^{\prime}(x, y)}
$$

From $F(x, y)=x$ we have that $\left(A_{1}+y^{2}\right)^{2}=\frac{b_{1}^{3} x^{7}}{\left(a_{2}+c_{2} y^{2}\right)^{2}+A_{1} x^{4}}$, which implies

$$
F_{x}^{\prime}(x, y)=\frac{4 x^{7} b_{1}^{3}\left(A_{1} x^{4}+2\left(c_{2} y^{2}+a_{2}\right)^{2}\right)}{\left(y^{2}+A_{1}\right)^{2}\left(A_{1} x^{4}+\left(c_{2} y^{2}+a_{2}\right)^{2}\right)^{2}}=8-\frac{4 A_{1} x^{4}}{\left(a_{2}+c_{2} y^{2}\right)^{2}+A_{1} x^{4}}>4
$$

Since $F_{y}^{\prime}(x, y)<0$, we get $x_{F}^{\prime}(y)>0$.

Theorem 14 If $a_{2} \leq A_{1} c_{2}$, then $T$ has no minimal period-two solution. If $a_{2}>A_{1} c_{2}$ and $T$ has a minimal period-two solution $\{(\Phi, \Psi), T(\Phi, \Psi)\}$, then $\{(\Phi, \Psi), T(\Phi, \Psi)\}$ is unstable. If $\mu_{1}$ and $\mu_{2}\left(\mu_{1}<\mu_{2}\right)$ are the eigenvalues of $J_{T^{2}}(\Phi, \Psi)$, then $\mu_{1}>0$ and $\mu_{2}>1$. All period-two solutions are ordered with respect to the north-east ordering. 
Proof If $a_{2} \leq A_{1} c_{2}$, the statement follows from Lemma 2. If $a_{2}>A_{1} c_{2}$, then from the first equation of (22) we have that

$$
\left(A_{1}+\Psi^{2}\right)^{2}=\frac{b_{1}^{3} \Phi^{7}}{\left(a_{2}+c_{2} \Psi^{2}\right)^{2}+A_{1} \Phi^{4}} .
$$

By substituting this into (24) we obtain

$$
\begin{aligned}
\operatorname{tr} J_{T^{2}}(\Phi, \Psi)= & \frac{4 \Psi\left(A_{1}+\Psi^{2}\right)\left(a_{2}\left(c_{2}^{2}\left(A_{1}+3 \Psi^{2}\right)+\Phi^{4}\right)+a_{2}^{2} c_{2}+c_{2}^{3} \Psi^{2}\left(A_{1}+2 \Psi^{2}\right)\right)}{b_{1}^{2} \Phi^{8}} \\
& +8-\frac{4 A_{1} \Phi^{4}}{\left(a_{2}+c_{2} \Psi^{2}\right)^{2}+A_{1} \Phi^{4}}>4 .
\end{aligned}
$$

The rest of the proof follows from the fact that $\operatorname{tr}_{T^{2}}(\Phi, \Psi)=\mu_{1}+\mu_{2}>4, \operatorname{det} J_{T^{2}}(\Phi, \Psi)=$ $\mu_{1} \mu_{2}>0$ and Lemma 6.

Theorem 15 If the map $T$ has a minimal period-two solution $\left\{\left(\Phi_{1}, \Psi_{1}\right), T\left(\Phi_{1}, \Psi_{1}\right)\right\}$, which is non-hyperbolic, then $D(p)=0$, where $D(p)$ is the discriminant of the polynomial

$$
p(y):=p_{22} y^{22}+p_{21} y^{21}+\cdots+p_{1} y+p_{0},
$$

where the coefficients $p_{i}, i=0, \ldots, 22$, are in the Appendix. If $\left\{\left(\Phi_{1}, \Psi_{1}\right), T\left(\Phi_{1}, \Psi_{1}\right)\right\}$ and $\left\{\left(\Phi_{2}, \Psi_{2}\right), T\left(\Phi_{2}, \Psi_{2}\right)\right\}$ are two minimal period-two solutions such that $T$ has no other minimal period-two solutions in $\llbracket\left(\Phi_{1}, \Psi_{1}\right),\left(\Phi_{2}, \Psi_{2}\right) \rrbracket=\left\{(x, y):\left(\Phi_{1}, \Psi_{1}\right) \preceq_{n e}(x, y) \preceq_{n e}\left(\Phi_{2}, \Psi_{2}\right)\right\}$ and $D(p) \neq 0$, then one of them is a saddle point and the other is a repeller.

Proof Period-two solution curves $\mathcal{C}_{F}=\{(x, y) \in \mathcal{R}: \tilde{F}(x, y)=0\}$ and $\mathcal{C}_{G}=\{(x, y) \in \mathcal{R}:$ $\tilde{G}(x, y)=0\}$, where

$$
\begin{aligned}
& \tilde{F}(x, y)=b_{1}^{3} x^{7}-A_{1} x^{4}\left(A_{1}+y^{2}\right)^{2}-\left(A_{1}+y^{2}\right)^{2}\left(a_{2}+c_{2} y^{2}\right)^{2} \text { and } \\
& \tilde{G}(x, y)=b_{1}^{2} y x^{8}-a_{2} x^{4}\left(A_{1}+y^{2}\right)^{2}-c_{2}\left(A_{1}+y^{2}\right)^{2}\left(a_{2}+c_{2} y^{2}\right)^{2}
\end{aligned}
$$

are algebraic curves. By using software Mathematica, one can see that the resultant of the polynomials $\tilde{F}(x, y)$ and $\tilde{G}(x, y)$ in variable $x$ is given by

$$
\begin{aligned}
R(\tilde{F}, \tilde{G}) & =-b_{1}^{6}\left(A_{1}+y^{2}\right)^{14}\left(a_{2}+c_{2} y^{2}\right)^{8}\left(-a_{2} b_{1}^{2}+2 A_{1} y^{3}+A_{1}^{2} y-b_{1}^{2} c_{2} y^{2}+y^{5}\right) p(y) \\
& =-b_{1}^{6}\left(A_{1}+y^{2}\right)^{14}\left(a_{2}+c_{2} y^{2}\right)^{8} \tilde{f}(y) p(y) .
\end{aligned}
$$

Suppose that $\left\{\left(\Phi_{1}, \Psi_{1}\right), T\left(\Phi_{1}, \Psi_{1}\right)\right\}$ is a non-hyperbolic minimal period-two solution. This implies that $\tilde{F}\left(\Phi_{1}, \Psi_{1}\right)=0, \tilde{G}\left(\Phi_{1}, \Psi_{1}\right)=0$. By Theorem 9.3 [28], $\tilde{F}$ and $\tilde{G}$ have a common non-constant factor if and only $R(\tilde{F}, \tilde{G})=0$, which implies that system $\tilde{F}(x, y)=0, \tilde{G}(x, y)=$ 0 has a solution if and only if $R(\tilde{F}, \tilde{G})=0$. Since $\tilde{f}\left(\Psi_{1}\right) \neq 0$, it must be $p\left(\Psi_{1}\right)=0$. Similarly 
as in Lemma 4, one can see that

$$
\begin{aligned}
x_{F}^{\prime}\left(\Psi_{1}\right)-x_{G}^{\prime}\left(\Psi_{1}\right) & =\frac{F_{y}^{\prime}\left(\Phi_{1}, \Psi_{1}\right)}{1-F_{x}^{\prime}\left(\Phi_{1}, \Psi_{1}\right)}-\frac{1-G_{y}^{\prime}\left(\Phi_{1}, \Psi_{1}\right)}{G_{x}^{\prime}\left(\Phi_{1}, \Psi_{1}\right)} \\
& =\frac{f_{1}}{1-e_{1}}-\frac{1-h_{1}}{g_{1}}=\frac{-1+\left(e_{1}+g_{1}\right)-\left(e_{1} h_{1}-f_{1} g_{1}\right)}{g_{1}\left(1-e_{1}\right)} \\
& =\frac{-p_{1}(1)}{g_{1}\left(1-e_{1}\right)}=\frac{\left(1-\mu_{1}\right)\left(1-\mu_{2}\right)}{g_{1}\left(e_{1}-1\right)},
\end{aligned}
$$

where $p_{1}(\mu)$ is the characteristic equation of the matrix

$$
J_{T^{2}}\left(\Phi_{1}, \Psi_{1}\right)=\left(\begin{array}{ll}
e_{1} & f_{1} \\
g_{1} & h_{1}
\end{array}\right)
$$

From Theorem 14 we have that $0<\mu_{1}<\mu_{2}$ and $\mu_{2}>1$. Since $\left\{\left(\Phi_{1}, \Psi_{1}\right), T\left(\Phi_{1}, \Psi_{1}\right)\right\}$ is nonhyperbolic, we obtain that $\mu_{1}=1$, from which it follows that $x_{F}^{\prime}\left(\Psi_{1}\right)-x_{G}^{\prime}\left(\Psi_{1}\right)=0$. Since $R(\tilde{F}, \tilde{G}) \not \equiv 0$, we have that $\mathcal{C}_{F}$ and $\mathcal{C}_{G}$ have no common component. By Lemmas 6 and 7 , from [27], the curves $\mathcal{C}_{F}$ and $\mathcal{C}_{G}$ intersect transversally at $\left(\Phi_{1}, \Psi_{1}\right)\left(\right.$ i.e., $\left.y_{\tilde{F}}^{\prime}\left(\Psi_{1}\right)-y_{\tilde{G}}^{\prime}\left(\Psi_{1}\right) \neq 0\right)$ if and only if $\Psi_{1}$ is zero of $p(y)$ of multiplicity one. By Theorem 9.4 [28], $p(y)$ has zeros of multiplicity greater than one if and only if the discriminant $D(p)$ of the polynomial $p(y)$ is equal to zero, which proves the first statement of the lemma.

Assume that $\left\{\left(\Phi_{1}, \Psi_{1}\right), T\left(\Phi_{1}, \Psi_{1}\right)\right\}$ and $\left\{\left(\Phi_{2}, \Psi_{2}\right), T\left(\Phi_{2}, \Psi_{2}\right)\right\}$ are two minimal period-two solutions such that $T$ has no other minimal period-two solutions in $\llbracket\left(\Phi_{1}, \Psi_{1}\right),\left(\Phi_{2}, \Psi_{2}\right) \rrbracket=$ $\left\{(x, y):\left(\Phi_{1}, \Psi_{1}\right) \preceq_{n e}(x, y) \preceq_{n e}\left(\Phi_{2}, \Psi_{2}\right)\right\}$ and $D(p) \neq 0$. From the previous discussion we have $x_{F}^{\prime}\left(\Psi_{i}\right)-x_{G}^{\prime}\left(\Psi_{i}\right) \neq 0, i=1,2$. Since $x_{F}\left(\Psi_{i}\right)-x_{G}\left(\Psi_{i}\right)=0, i=1,2$, it follows that $\left(x_{F}^{\prime}\left(\Psi_{1}\right)-\right.$ $\left.x_{G}^{\prime}\left(\Psi_{1}\right)\right)\left(x_{F}^{\prime}\left(\Psi_{2}\right)-x_{G}^{\prime}\left(\Psi_{2}\right)\right)<0$. Indeed assume, for example, that $x_{F}^{\prime}\left(\Psi_{1}\right)-x_{G}^{\prime}\left(\Psi_{1}\right)<0$ and $x_{F}^{\prime}\left(\Psi_{2}\right)-x_{G}^{\prime}\left(\Psi_{2}\right)<0$. Then there exists $\epsilon>0$ such that $x_{F}(y)-x_{G}(y)<0$ for $y \in\left(\Psi_{1}, \Psi_{1}+\epsilon\right)$ and $x_{F}(y)-x_{G}(y)>0$ for $y \in\left(\Psi_{2}-\epsilon, \Psi_{2}\right)$. Since $x_{F}(y)-x_{G}(y)$ is a continuous function, this implies that there exists $\Psi \in\left(\Psi_{1}, \Psi_{2}\right)$ such that $x_{F}(\Psi)-x_{G}(\Psi)=0$, which is a contradiction. The rest of the proof follows from the fact that $e_{i}>4$ and $g_{i}<0, i=1,2$.

Notice that

$$
D(p)=-\frac{1}{p_{22}} R\left(p, p^{\prime}\right),
$$

where $R\left(p, p^{\prime}\right)=\operatorname{det} \operatorname{Syl}\left(p, p^{\prime}\right)$, the determinant of the $\operatorname{Sylvester}$ matrix $\operatorname{Syl}\left(p, p^{\prime}\right)$, see [28, $29]$, and

$$
\operatorname{Syl}\left(p, p^{\prime}\right)=\left(\begin{array}{cccccccc}
p_{22} & p_{21} & \ldots & p_{1} & p_{0} & 0 & \cdots & 0 \\
0 & p_{22} & p_{21} & \cdots & p_{1} & p_{0} & \cdots & 0 \\
\vdots & & & & & & & \\
0 & \ldots & p_{22} & p_{21} & p_{20} & \ldots & p_{1} & p_{0} \\
22 p_{21} & 21 p_{20} & \ldots & p_{1} & 0 & 0 & \cdots & 0 \\
0 & 22 p_{21} & 21 p_{20} & \cdots & p_{1} & 0 & \cdots & 0 \\
\vdots & & & & & & & \\
0 & 0 & \ldots & 22 p_{21} & 21 p_{20} & 20 p_{19} & \cdots & p_{1}
\end{array}\right) .
$$


Theorem 16 If $a_{2}>A_{1} c_{2}$ and $\Gamma(\bar{y})<0$, then $T$ has one equilibrium point $E(\bar{x}, \bar{y})$, which is a repeller, and there exists at least one minimal period-two solution $\{(\psi, \phi), T(\psi, \phi)\}$ which is non-hyperbolic or a saddle point. If $T$ has no minimal period-two solutions which are non-hyperbolic, then $(\psi, \phi) \ll_{n e} E \ll_{n e} T(\psi, \phi)$.

Proof By Theorem 10 we have that $T$ has one equilibrium point $E(\bar{x}, \bar{y})$, which is a repeller. This and Lemma 2 imply that $T^{n}\left(x_{0}, y_{0}\right)$ is asymptotic to either $(0, \infty)$ or $(\infty, 0)$, or a minimal period-two solution, for all $\left(x_{0}, y_{0}\right) \in \mathcal{R}$. Let $\mathcal{B}(0, \infty)$ be the basin of attraction of $(0, \infty)$, and let $\mathcal{B}(\infty, 0)$ be the basin of attraction of $(\infty, 0)$. By using Theorem 9 one can prove that $\operatorname{int}\left(Q_{2}(E)\right) \subset \mathcal{B}(0, \infty)$ and $\operatorname{int}\left(Q_{4}(E)\right) \subset \mathcal{B}(\infty, 0)$. Let $\mathcal{S}_{1}$ denote the boundary of $\mathcal{B}(\infty, 0)$ considered as a subset of $Q_{1}(E)$, and let $\mathcal{S}_{2}$ denote the boundary of $\mathcal{B}(\infty, 0)$ considered as a subset of $Q_{3}(E)$. It is easy to see that $E \in \mathcal{S}_{1}, E \in \mathcal{S}_{2}$ and $T(\mathcal{R}) \subset \operatorname{int}(\mathcal{R})$. Now we prove the following claim.

Claim 1 Let $\mathcal{S}_{1}$ and $\mathcal{S}_{2}$ be the sets defined as above. Then

(a) If $\left(x_{0}, y_{0}\right) \in \mathcal{B}(\infty, 0)$, then $\left(x_{1}, y_{1}\right) \in \mathcal{B}(\infty, 0)$ for all $\left(x_{0}, y_{0}\right) \preceq_{\text {se }}\left(x_{1}, y_{1}\right)$.

(b) If $\left(x_{0}, y_{0}\right) \in \mathcal{S}_{1} \cup \mathcal{S}_{2}$, then $\left(x_{1}, y_{1}\right) \in \operatorname{int}(\mathcal{B})(\infty, 0)$ for all $\left(x_{0}, y_{0}\right) \ll_{\text {se }}\left(x_{1}, y_{1}\right)$.

(c) $\mathcal{S}_{1} \cap \operatorname{int}\left(Q_{1}(E)\right) \neq \emptyset$ and $\mathcal{S}_{2} \cap \operatorname{int}\left(Q_{3}(E)\right) \neq \emptyset$.

(d) $T\left(\mathcal{S}_{1} \cup \mathcal{S}_{2}\right) \subseteq \mathcal{S}_{1} \cup \mathcal{S}_{2}$.

(e) $\left(x_{0}, y_{0}\right),\left(x_{1}, y_{1}\right) \in \mathcal{S}_{1} \cup \mathcal{S}_{2} \Rightarrow\left(x_{0}, y_{0}\right) \ll_{n e}\left(x_{1}, y_{1}\right)$ or $\left(x_{1}, y_{1}\right) \ll_{n e}\left(x_{0}, y_{0}\right)$.

Proof (a) The statement follows from $T^{n}\left(x_{0}, y_{0}\right) \preceq_{s e} T^{n}\left(x_{1}, y_{1}\right) \preceq_{s e}(\infty, 0)$ and $T^{n}\left(x_{0}, y_{0}\right) \rightarrow$ $(\infty, 0)$ as $n \rightarrow \infty$.

(b) The claim (b) follows from the observation that there exists a ball centered at $\left(x_{0}, y_{0}\right)$ with the property that all its points $(x, y)$ satisfy $(x, y) \ll_{s e}\left(x_{1}, y_{1}\right)$. But one of these points necessarily lies in $\mathcal{B}(\infty, 0)$, so by (a) there exists $\left(x_{1}, y_{1}\right) \in \mathcal{B}(\infty, 0)$. Furthermore, there exists a ball centered at $\left(x_{1}, y_{1}\right)$ with the property that all its points $(x, y)$ satisfy $(x, y) \in \mathcal{B}(\infty, 0)$, which implies $\left(x_{1}, y_{1}\right) \in \operatorname{int}(\mathcal{B})(\infty, 0)$.

(c) Take $y^{\prime}>\bar{y}$ arbitrary (but fixed). Since $T$ is strongly competitive, we have $T\left(\bar{x}, y^{\prime}\right) \ll_{\text {se }}$ $T(\bar{x}, \bar{y})$, which implies $T\left(\bar{x}, y^{\prime}\right) \in \operatorname{int}\left(Q_{2}(E)\right)$. This implies that there exists a ball $B_{\varepsilon}\left(T\left(\bar{x}, y^{\prime}\right)\right)$ with the property $B_{\varepsilon}\left(T\left(\bar{x}, y^{\prime}\right)\right) \subset \operatorname{int}\left(Q_{2}(E)\right)$. Since $T$ is a continuous map on a set $\mathbb{R}_{+}^{2} \backslash$ $\{(0, y): y \geq 0\}$, then there exists a ball $B_{\delta_{1}}\left(\bar{x}, y^{\prime}\right)$ such that $T\left(B_{\delta_{1}}\left(\bar{x}, y^{\prime}\right)\right) \subset B_{\varepsilon}\left(T\left(\bar{x}, y^{\prime}\right)\right) \subset$ $\operatorname{int}\left(Q_{2}(E)\right)$, which implies $T^{n}(x, y) \rightarrow(0, \infty)$ as $n \rightarrow \infty$ for all $(x, y) \in B_{\delta_{1}}\left(\bar{x}, y^{\prime}\right)$. Similarly, one can prove that then there exists a ball $B_{\delta_{2}}\left(\bar{x}+\delta_{1} / 2, \bar{y}\right)$ such that $T^{n}(x, y) \rightarrow(\infty, 0)$ as $n \rightarrow \infty$ for all $(x, y) \in B_{\delta_{2}}\left(\bar{x}+\delta_{1} / 2, \bar{y}\right)$. Let $y^{\prime \prime}=\sup \left\{y: \lim _{n \rightarrow \infty} T^{n}\left(\bar{x}+\delta_{1} / 2, y\right)=(\infty, 0)\right\}$. It is easy to see that $\left(\bar{x}+\delta_{1} / 2, y^{\prime \prime}\right) \in \mathcal{S}_{1} \cap \operatorname{int}\left(Q_{1}(E)\right)$. The assertion concerning $\mathcal{S}_{2}$ is proved in a similar fashion.

(d) Take $(x, y) \in \mathcal{S}_{1} \cup \mathcal{S}_{2}$. Assume that $T(x, y) \notin \mathcal{S}_{1} \cup \mathcal{S}_{2}$. Since $\mathcal{S}_{1} \cup \mathcal{S}_{2}=\partial \mathcal{B}(\infty, 0)=$ $\overline{\mathcal{B}(\infty, 0)} \backslash \operatorname{int}(\mathcal{B}(\infty, 0))$, then either $T(x, y) \in \operatorname{int}(\mathcal{B}(\infty, 0))$ or $T(x, y) \notin \overline{\mathcal{B}(\infty, 0)}$. Assume that $T(x, y) \in \operatorname{int}(\mathcal{B}(\infty, 0))$. This implies that there exists a ball $B_{\varepsilon}(T(x, y))$ with the property $B_{\varepsilon}(T(x, y)) \subset \operatorname{int}(\mathcal{B}(\infty, 0))$. Since $T$ is a continuous map on the set $\mathbb{R}_{+}^{2} \backslash\{(0, y): y \geq 0\}$, then there exists a ball $B_{\delta}(x, y), \delta>0$ such that $T\left(B_{\delta}(x, y)\right) \subset B_{\varepsilon}(T(x, y))$, which implies $B_{\delta}(x, y) \subset$ $\mathcal{B}(\infty, 0)$. This is in contradiction with $(x, y) \in \mathcal{S}_{1} \cup \mathcal{S}_{2}=\partial \mathcal{B}(\infty, 0)$. Hence $T(x, y) \in \mathcal{S}_{1} \cup \mathcal{S}_{2}$ in this case. Similarly, one can prove that $T(x, y) \in \mathcal{S}_{1} \cup \mathcal{S}_{2}$ if $T(x, y) \notin \overline{\mathcal{B}(\infty, 0)}$. This implies that $T\left(\mathcal{S}_{1} \cup \mathcal{S}_{2}\right) \subseteq\left(\mathcal{S}_{1} \cup \mathcal{S}_{2}\right)$. 
(e) Assume that $\left(x_{0}, y_{0}\right),\left(x_{1}, y_{1}\right) \in \mathcal{S}_{1} \cup \mathcal{S}_{2} \Rightarrow\left(x_{0}, y_{0}\right) \preceq_{s e}\left(x_{1}, y_{1}\right)$ and $\left(x_{0}, y_{0}\right) \neq\left(x_{1}, y_{1}\right)$. Since $T$ is strongly competitive, we get $T\left(x_{0}, y_{0}\right) \ll_{n e} T\left(x_{1}, y_{1}\right)$. This contradicts (e) and (b), which completes the proof.

In view of Claim 1 , we have that $\left(\mathcal{S}_{1} \cup \mathcal{S}_{2}, \ll_{n e}\right)$ is a totally ordered set which is invariant under $T$. If $\left(x_{0}, y_{0}\right) \in \mathcal{S}_{1} \cup \mathcal{S}_{2}$, then $\left\{T^{(2 n)}\left(x_{0}, y_{0}\right)\right\}$ is eventually component-wise monotone. Then there exists a minimal period-two solution $\{(\Phi, \Psi), T(\Phi, \Psi)\} \in \mathcal{S}_{1} \cup \mathcal{S}_{2} \subset Q_{1}(E) \cup$ $Q_{3}(E)$ such that $T^{(2 n)}\left(x_{0}, y_{0}\right) \rightarrow(\Phi, \Psi)$ as $n \rightarrow \infty$. By Theorem 14, $\{(\Phi, \Psi), T(\Phi, \Psi)\}$ is a non-hyperbolic or a saddle point. Assume that $T$ has no minimal period-two solutions which are non-hyperbolic points and, for example, that $(\Phi, \Psi), T(\Phi, \Psi) \in \mathcal{S}_{1}$ such that $E \ll_{n e}(\Phi, \Psi) \ll_{n e} T(\Phi, \Psi)$. Since $\{(\Phi, \Psi), T(\Phi, \Psi)\}$ is a saddle point, in view of Theorems 6, 7 and 8, we have that the global stable manifolds $\mathcal{W}^{s}(\{(\Phi, \Psi), T(\Phi, \Psi)\})$ are the union of two curves $\mathcal{W}^{s}(\Phi, \Psi)$ and $\mathcal{W}^{s}(T(\Phi, \Psi))$ whose endpoints are repeller points such that $T\left(\mathcal{W}^{s}(\Phi, \Psi)\right)=\mathcal{W}^{s}(T(\Phi, \Psi))$ and $E \ll_{n e} \overline{\mathcal{W}^{s}(\Phi, \Psi)} \ll_{n e} \overline{\mathcal{W}^{s}(T(\Phi, \Psi))}$. If $P_{1}$ and $P_{2}\left(P_{1} \preceq_{n e} P_{2}\right)$ are endpoints of $\mathcal{W}^{s}(\Phi, \Psi)$, then $T\left(P_{1}\right)$ and $T\left(P_{2}\right)$ are endpoints of $T\left(\mathcal{W}^{s}(\Phi, \Psi)\right)$ and either $T\left(P_{1}\right) \preceq_{n} T\left(P_{2}\right)$ or $T\left(P_{2}\right) \preceq_{n e} T\left(P_{1}\right)$. Assume, for example, that $P_{1} \ll_{n e} \mathcal{W}^{s}(\Phi, \Psi) \ll_{n e} P_{2} \ll_{n e} T\left(P_{2}\right) \ll_{n e} \mathcal{W}^{s}(T(\Phi, \Psi)) \ll_{n e} T\left(P_{1}\right)$. By Theorem 15 between two repellers $P_{2}$ and $T\left(P_{2}\right)$, there exists a saddle point $S_{1}$ where its stable manifold is the union of two invariant curves $\mathcal{W}^{s}\left(S_{1}\right)$ and $\mathcal{W}^{s}\left(T\left(S_{1}\right)\right)$ whose endpoints are repellers such that $P_{2} \ll_{n e} \overline{\mathcal{W}^{s}\left(S_{1}\right)} \ll_{n e} T\left(P_{2}\right)$. Continuing in this way, we obtain that $T$ has infinitely many minimal period-two solutions $\left\{P_{i}, T\left(P_{i}\right)\right\}$, which is in contradiction with the fact that $T$ has at most eleven minimal period-two solutions. Hence $(\Phi, \Psi) \ll_{n e} E \ll_{n e} T(\Phi, \Psi)$.

Corollary 2 Assume that $a_{2}>A_{1} c_{2}, \Gamma(\bar{y})<0$ and $D(p) \neq 0$. Then there exists one equilibrium point $E$ which is a repeller. Further, the set $\operatorname{int}\left(Q_{1}(E)\right) \cup \operatorname{int}\left(Q_{3}(E)\right)$ contains an odd number of minimal period-two solutions $\left\{\left(\Phi_{i}, \Psi_{i}\right),\left(\tilde{\Phi}_{i}, \tilde{\Psi}_{i}\right)\right\}, i=1, \ldots, 2 n+1$, such that $\left(\Phi_{i+1}, \Psi_{i+1}\right) \ll_{n e}\left(\Phi_{i}, \Psi_{i}\right) \ll_{n e} E$ and $E \ll_{n e}\left(\tilde{\Phi}_{i}, \tilde{\Psi}_{i}\right) \ll_{n e}\left(\tilde{\Phi}_{i+1}, \tilde{\Psi}_{i+1}\right)$, where $\left(\tilde{\Phi}_{i}, \tilde{\Psi}_{i}\right)=$ $T\left(\Phi_{i}, \Psi_{i}\right)$. Furthermore, odd indexed period-two points are saddles and even indexed period-two points are repellers.

Proof By Theorem 10 we have that $T$ has one equilibrium point $E(\bar{x}, \bar{y})$, which is a repeller. By Theorem 15 all minimal period-two solutions are hyperbolic and the number of minimal period-two solutions is finite. In view of Claim 1, $T$ has at least one minimal period-two solution which is a saddle point. Let $\left\{P_{1}, T\left(P_{1}\right)\right\}$ be a minimal periodtwo solution which is a saddle point such that $P_{1} \ll_{n e} E \ll_{n e} T\left(P_{1}\right)$ and $T$ has no minimal period-two solutions in $\llbracket E, T\left(P_{1}\right) \rrbracket$ and $\llbracket P_{1}, E \rrbracket$. Such a minimal period-two solution exists in view of Theorem 15. The map $T^{2}$ satisfies all conditions of Theorems 6, 7 and 8, which yields the existence of the global stable manifolds $\mathcal{W}^{s}\left(\left\{P_{1}, \tilde{P}_{1}\right\}\right)$ which are the union of two curves $\mathcal{W}^{s}\left(P_{1}\right)$ and $\mathcal{W}^{s}\left(\tilde{P}_{1}\right)$ that have a common endpoint $E$. If $T$ has minimal period-two solutions in $\operatorname{int}\left(Q_{1}\left(T\left(P_{1}\right)\right)\right) \cup \operatorname{int}\left(Q_{3}\left(P_{1}\right)\right)$, let $\left\{P_{2}, \tilde{P}_{2}\right\}\left(P_{2} \ll_{n e} \tilde{P}_{2}\right)$ denote minimal periodtwo solutions such that $T$ has no other minimal period-two solutions in $\llbracket T\left(P_{1}\right), T\left(P_{2}\right) \rrbracket$ and $\llbracket P_{2}, P_{1} \rrbracket$. Then $\mathcal{W}^{s}\left(P_{1}\right)$ has the second endpoint at $P_{2}$ and $\mathcal{W}^{s}\left(T\left(P_{1}\right)\right)$ has the second endpoint at $T\left(P_{2}\right)$ and $P_{2} \ll_{n e} P_{1} \ll_{n e} E \ll_{n e} T\left(P_{1}\right) \ll_{n e} T\left(P_{2}\right)$. Furthermore, a minimal period-two solution $\left\{P_{2}, T\left(P_{2}\right)\right\}$ is a repeller. Similarly as in Theorem 16, one can prove that $\operatorname{int}\left(Q_{1}\left(T\left(P_{2}\right)\right)\right) \cup \operatorname{int}\left(Q_{3}\left(P_{2}\right)\right)$ contains at least one minimal period-two solution which 
is a saddle point. Since the number of minimal period-two solutions is finite, continuing in this way, we will end with a minimal period-two solution which is a saddle point, from which the proof follows.

Corollary 3 If $a_{2}>A_{1} c_{2}$ and $\Gamma(\bar{y})>0$, then there exists one equilibrium point $E$ which is a saddle point. If $D(p) \neq 0$, then $\operatorname{int}\left(Q_{1}(E)\right) \cup \operatorname{int}\left(Q_{3}(E)\right)$ contains an even number of minimal period-two solutions $\left\{\left(\Phi_{i}, \Psi_{i}\right),\left(\tilde{\Phi}_{i}, \tilde{\Psi}_{i}\right)\right\}, i=1, \ldots, 2 n$, such that $\left(\Phi_{i+1}, \Psi_{i+1}\right) \ll_{n e}\left(\Phi_{i}, \Psi_{i}\right) \ll_{n e}$ $E$ and $E \ll_{n e}\left(\tilde{\Phi}_{i}, \tilde{\Psi}_{i}\right) \ll_{n e}\left(\tilde{\Phi}_{i+1}, \tilde{\Psi}_{i+1}\right)$ and $\left(\tilde{\Phi}_{i}, \tilde{\Psi}_{i}\right)=T\left(\Phi_{i}, \Psi_{i}\right)$. Furthermore, even indexed period-two points are saddles and odd indexed period-two points are repellers.

Proof The proof is similar as the proof of Corollary 2 and it will be omitted.

Based on a series of numerical simulations, we propose the following conjecture.

Conjecture 1 System (1) has at most one minimal period-two solution.

\section{Global behavior}

In this section we present global dynamics of system (1) in different parametric regions. We have five parametric regions with different dynamics which will be characterized by the following five theorems.

\subsection{The case where the equilibrium points and period-two solutions are hyperbolic points $\left(\Delta_{1} \neq 0\right.$ and $\left.D(p) \neq 0\right)$}

Theorem 17 Assume that $\Delta_{1}<0$. Then system (1) has three equilibrium solutions $E_{1} \ll_{n e}$ $E_{2} \ll_{n e} E_{3}$, where $E_{1}$ and $E_{3}$ are saddle points and $E_{2}$ is a repeller. In this case there exist four invariant continuous curves $\mathcal{W}^{s}\left(E_{1}\right), \mathcal{W}^{s}\left(E_{3}\right), \mathcal{W}^{u}\left(E_{1}\right), \mathcal{W}^{u}\left(E_{3}\right)$, where $\mathcal{W}^{s}\left(E_{1}\right), \mathcal{W}^{s}\left(E_{3}\right)$ have end points at $E_{2}$, and are graphs of increasing functions. The curves $\mathcal{W}^{u}\left(E_{1}\right), \mathcal{W}^{u}\left(E_{3}\right)$ are the graphs of decreasing functions. Every solution $\left\{\left(x_{n}, y_{n}\right)\right\}$ which starts below $\mathcal{W}^{s}\left(E_{1}\right) \cup$ $\mathcal{W}^{s}\left(E_{3}\right)$ in the south-east ordering is asymptotic to $(0, \infty)$, and every solution $\left\{\left(x_{n}, y_{n}\right)\right\}$ which starts above $\mathcal{W}^{s}\left(E_{1}\right) \cup \mathcal{W}^{s}\left(E_{3}\right)$ in the south-east ordering is asymptotic to $(\infty, 0)$. The first quadrant of initial condition $Q_{1}=\left\{\left(x_{0}, y_{0}\right): x_{0}>0, y_{0} \geq 0\right\}$ is the union of five disjoint basins of attraction, i.e.,

$$
Q_{1}=\mathcal{B}(0, \infty) \cup \mathcal{B}(\infty, 0) \cup \mathcal{B}\left(E_{1}\right) \cup \mathcal{B}\left(E_{3}\right) \cup \mathcal{B}\left(E_{2}\right),
$$

where $\mathcal{B}\left(E_{2}\right)=\left\{E_{2}\right\}, \mathcal{B}\left(E_{1}\right)=\mathcal{W}^{s}\left(E_{1}\right), \mathcal{B}\left(E_{3}\right)=\mathcal{W}^{s}\left(E_{3}\right)$ and

$$
\begin{aligned}
& \mathcal{B}(0, \infty)=\left\{(x, y) \mid(x, y) \preceq_{s e}\left(\tilde{x}_{0}, \tilde{y}_{0}\right) \text { for some }\left(\tilde{x}_{0}, \tilde{y}_{0}\right) \in \mathcal{W}^{s}\left(E_{1}\right) \cup \mathcal{W}^{s}\left(E_{3}\right)\right\}, \\
& \mathcal{B}(\infty, 0)=\left\{(x, y) \mid\left(\tilde{x}_{1}, \tilde{y}_{1}\right) \preceq_{s e}(x, y) \text { for some }\left(\tilde{x}_{1}, \tilde{y}_{1}\right) \in \mathcal{W}^{s}\left(E_{1}\right) \cup \mathcal{W}^{s}\left(E_{3}\right)\right\} .
\end{aligned}
$$

Proof Theorem 11 implies that there exist three equilibrium points, namely $E_{1}, E_{2}$ and $E_{3}$, such that $E_{1} \ll_{n e} E_{2} \ll_{n e} E_{3}$. In this case, $E_{1}$ and $E_{3}$ are saddle points and $E_{2}$ is a repeller. In view of (17), the map $T$ is competitive on $\mathcal{R}$ and strongly competitive on $\operatorname{int}(\mathcal{R})$. It follows from the Perron-Frobenius theorem and a change of variables [14] that at each point the Jacobian matrix of a strongly competitive map has two real and distinct eigenvalues, the larger one in absolute value being positive, and that corresponding eigenvectors may be 
chosen to point in the direction of the second and first quadrant, respectively. Also, one can show that if the map is strongly competitive, then no eigenvector is aligned with a coordinate axis.

Since $\Delta_{1}<0$ implies $a_{2}<A_{1} c_{2}$, we have that $\operatorname{det} J_{T}\left(E_{i}\right)>0, i=1,3$. Hence, all conditions of Theorems 6, 7 and 8 are satisfied, which yields the existence of the global stable manifolds $\mathcal{W}^{s}\left(E_{1}\right), \mathcal{W}^{s}\left(E_{3}\right)$ and the global unstable manifolds $\mathcal{W}^{u}\left(E_{1}\right), \mathcal{W}^{u}\left(E_{3}\right)$, where $\mathcal{W}^{s}\left(E_{1}\right), \mathcal{W}^{s}\left(E_{3}\right)$ are passing through the point $E_{2}$ and are graphs of increasing functions. The curves $\mathcal{W}^{u}\left(E_{1}\right), \mathcal{W}^{u}\left(E_{3}\right)$ are the graphs of decreasing functions. Let

$$
\begin{aligned}
& \mathcal{W}^{-}=\left\{(x, y) \mid(x, y) \preceq_{\text {se }}\left(\tilde{x}_{0}, \tilde{y}_{0}\right) \text { for some }\left(\tilde{x}_{0}, \tilde{y}_{0}\right) \in \mathcal{W}^{s}\left(E_{1}\right) \cup \mathcal{W}^{s}\left(E_{3}\right)\right\}, \\
& \mathcal{W}^{+}=\left\{(x, y) \mid\left(\tilde{x}_{1}, \tilde{y}_{1}\right) \preceq_{s e}(x, y) \text { for some }\left(\tilde{x}_{1}, \tilde{y}_{1}\right) \in \mathcal{W}^{s}\left(E_{1}\right) \cup \mathcal{W}^{s}\left(E_{3}\right)\right\} .
\end{aligned}
$$

Take $\left(x_{0}, y_{0}\right) \in \mathcal{W}^{-} \cap \mathcal{R}$. By Theorem 7 we have that there exists $n_{0}>0$ such that $T^{n}\left(x_{0}, y_{0}\right) \in \operatorname{int}\left(\left(Q_{2}\left(E_{1}\right) \cup Q_{2}\left(E_{3}\right)\right) \cap \mathcal{R}\right), n>n_{0}$. In view of Theorem 9, since $E_{1}$ and $E_{3}$ are saddle points, we obtain that for all $\left(x_{0}, y_{0}\right) \in \operatorname{int}\left(\left(Q_{2}\left(E_{1}\right) \cup Q_{2}\left(E_{3}\right)\right) \cap \mathcal{R}\right)$, there exists a subsolution $\left(u_{0}, v_{0}\right)$ such that $\left(x_{0}, y_{0}\right) \preceq_{s e}\left(u_{0}, v_{0}\right)$. By monotonicity we have that $(0, \infty) \preceq_{s e}$ $T^{n}\left(x_{0}, y_{0}\right) \preceq_{s e} T^{n}\left(u_{0}, v_{0}\right) \ll E_{3}$ if $\left(x_{0}, y_{0}\right) \in \operatorname{int}\left(Q_{2}\left(E_{3}\right) \cap \mathcal{R}\right)$ and $(0, \infty) \preceq_{s e} T^{n}\left(x_{0}, y_{0}\right) \preceq_{s e}$ $T^{n}\left(u_{0}, v_{0}\right) \ll E_{1}$ if $\left(x_{0}, y_{0}\right) \in \operatorname{int}\left(Q_{2}\left(E_{1}\right) \cap \mathcal{R}\right)$. For the sequence $\left(u_{n}, v_{n}\right)=T^{n}\left(u_{0}, v_{0}\right)$, we have $\left(u_{n+1}, v_{n+1}\right) \preceq_{s e}\left(u_{n}, v_{n}\right) \preceq_{s e}\left(u_{0}, v_{0}\right)$. The sequence $\left\{u_{n}\right\}$ is monotone decreasing and bounded from below and $\left\{v_{n}\right\}$ is monotone increasing. Since $T$ has no other equilibrium point except $E_{1}, E_{2}$ and $E_{3}$, this implies that the sequence $\left\{u_{n}\right\}$ has finite limit and $v_{n} \rightarrow+\infty$ as $n \rightarrow \infty$. From $u_{n+1}=\left(b_{1} u_{n}^{2}\right) /\left(A_{1}+v_{n}^{2}\right)$ we obtain that $u_{n} \rightarrow 0$ as $n \rightarrow \infty$. This implies $T^{n}\left(x_{0}, y_{0}\right) \rightarrow(0, \infty)$ as $n \rightarrow \infty$. If $\left(x_{0}, y_{0}\right) \in \mathcal{W}^{+} \cap \mathcal{R}$, the proof is similar and we skip it.

Another way of completing the proof is by using (3) of Lemma 2.

Theorem 18 Assume that $\Delta_{1}>0$. If

$$
a_{2} \leq A_{1} c_{2}
$$

or

$a_{2}>A_{1} c_{2}$ and $\Gamma(\bar{y})>0$ and $T$ has no minimal period-two solution,

then system (1) has one equilibrium solution $E(\bar{x}, \bar{y})$, which is a saddle point. There exists the global stable manifold $\mathcal{W}^{s}(E)$ which is the graph of a continuous increasing function and the global unstable manifold $\mathcal{W}^{u}(E)$, which is the graph of a continuous decreasing function. Every solution $\left\{\left(x_{n}, y_{n}\right)\right\}$ which starts below $\mathcal{W}^{s}(E)$ in the south-east ordering is asymptotic to $(0, \infty)$, and every solution $\left\{\left(x_{n}, y_{n}\right)\right\}$ which starts above $\mathcal{W}^{s}(E)$ in the south-east ordering is asymptotic to $(\infty, 0)$. The first quadrant of initial condition $Q_{1}=\left\{\left(x_{0}, y_{0}\right): x_{0}>0, y_{0} \geq 0\right\}$ is the union of three disjoint basins of attraction, $Q_{1}=\mathcal{B}(0, \infty) \cup \mathcal{B}(\infty, 0) \cup \mathcal{B}(E)$, where $\mathcal{B}(E)=\mathcal{W}^{s}(E)$ and

$$
\begin{aligned}
& \mathcal{B}(0, \infty)=\left\{(x, y) \mid(x, y) \preceq_{s e}\left(\tilde{x}_{0}, \tilde{y}_{0}\right) \text { for some }\left(\tilde{x}_{0}, \tilde{y}_{0}\right) \in \mathcal{W}^{s}(E)\right\} ; \\
& \mathcal{B}(\infty, 0)=\left\{(x, y) \mid\left(\tilde{x}_{1}, \tilde{y}_{1}\right) \preceq_{\text {se }}(x, y) \text { for some }\left(\tilde{x}_{1}, \tilde{y}_{1}\right) \in \mathcal{W}^{s}(E)\right\} .
\end{aligned}
$$




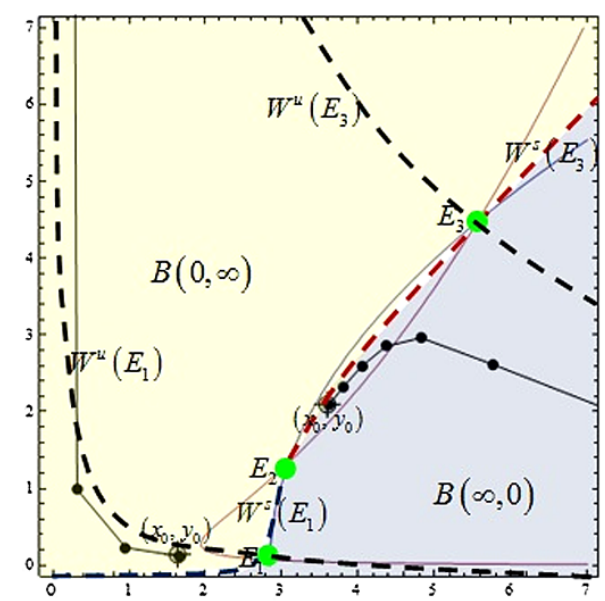

a)

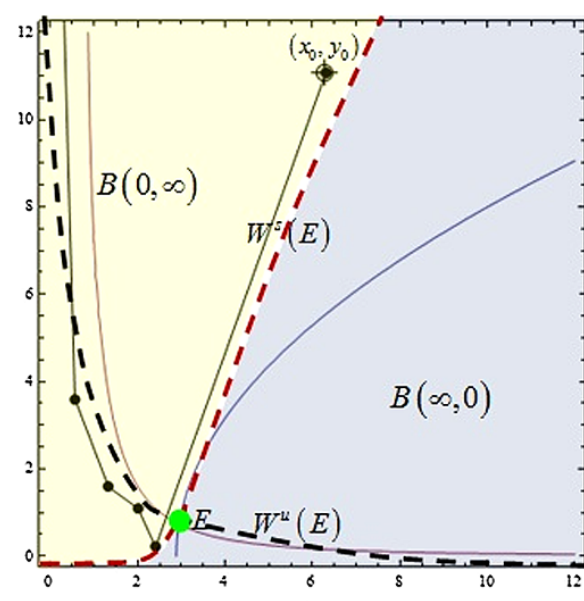

b)

Figure 1 (a) Visual illustration of Theorem 17. (b) Visual illustration of Theorem 18.

Proof The conditions of this theorem and $a_{2} \neq A_{1} c_{2}$ imply all assumptions of Theorems 6, 7 and 8 for $\mathcal{R}$. The proof of this theorem in this case is similar to the proof of Theorem 17 and will be skipped.

Now, we assume that $a_{2}=A_{1} c_{2}$. In view of Lemma 2 , the set

$$
\mathcal{I}=\left\{\left(x, \sqrt{\frac{b_{1} x^{2}-A_{1} \bar{x}}{\bar{x}}}\right): x \geq \sqrt{\frac{A_{1} \bar{x}}{b_{1}}}\right\}
$$

is invariant and contains equilibrium $E$, and $T(x, y)=E$ for $(x, y) \in \mathcal{I}$. In view of the uniqueness of the global stable manifold, we conclude that $\mathcal{W}^{s}(E)=\mathcal{I}$. Let

$$
\begin{aligned}
& \mathcal{W}^{-}=\left\{(x, y) \mid(x, y) \preceq_{s e}\left(\tilde{x}_{0}, \tilde{y}_{0}\right) \text { for some }\left(\tilde{x}_{0}, \tilde{y}_{0}\right) \in \mathcal{W}^{s}(E)\right\} ; \\
& \mathcal{W}^{+}=\left\{(x, y) \mid\left(\tilde{x}_{1}, \tilde{y}_{1}\right) \preceq_{s e}(x, y) \text { for some }\left(\tilde{x}_{1}, \tilde{y}_{1}\right) \in \mathcal{W}^{s}(E)\right\} .
\end{aligned}
$$

Take any point $\left(x_{0}, y_{0}\right) \in \mathcal{W}^{+}(E)$. Then there exists the point $\left(x_{l}, y_{l}\right) \in \mathcal{I}$ such that $\left(x_{l}, y_{l}\right) \ll_{s e}\left(x_{0}, y_{0}\right)$. Since the map $T$ is strongly competitive, then $E=T\left(x_{l}, y_{l}\right) \ll_{s e} T\left(x_{0}, y_{0}\right)$. This implies $T\left(x_{0}, y_{0}\right) \in \operatorname{int}\left(Q_{4}(E) \cap \mathcal{R}\right)$. If $\left(x_{0}, y_{0}\right) \in \mathcal{W}^{-}(E)$, then there exists the point $\left(x_{r}, y_{r}\right) \in \mathcal{I}$ such that $\left(x_{0}, y_{0}\right) \ll_{s e}\left(x_{r}, y_{r}\right)$. Since the map $T$ is strongly competitive, then $T\left(x_{0}, y_{0}\right) \ll_{s e} E=T\left(x_{r}, y_{r}\right)$. This implies $T\left(x_{0}, y_{0}\right) \in \operatorname{int}\left(Q_{2}(E) \cap \mathcal{R}\right)$. Similarly as in Theorem 17 , one can prove that $T^{n}\left(x_{0}, y_{0}\right) \rightarrow(0, \infty)$ as $n \rightarrow \infty$ if $\left(x_{0}, y_{0}\right) \in \operatorname{int}\left(Q_{2}(E) \cap \mathcal{R}\right)$ and $T^{n}\left(x_{0}, y_{0}\right) \rightarrow(\infty, 0)$ as $n \rightarrow \infty$ if $\left(x_{0}, y_{0}\right) \in \operatorname{int}\left(Q_{4}(E) \cap \mathcal{R}\right)$, from which the proof follows in this case.

See Figure 1 for visual illustration of Theorems 17 and 18.

Theorem 19 If $a_{2}>A_{1} c_{2}$ and $\Gamma(\bar{y})<0$, then there exists one equilibrium point $E$ which is a repeller. If $D(p) \neq 0$, then $\operatorname{int}\left(Q_{1}(E)\right) \cup \operatorname{int}\left(Q_{3}(E)\right)$ contains an odd number of minimal periodtwo solutions $\left\{\left(\Phi_{i}, \Psi_{i}\right),\left(\tilde{\Phi}_{i}, \tilde{\Psi}_{i}\right)\right\}, i=1, \ldots, 2 n+1$, such that $\left(\Phi_{i+1}, \Psi_{i+1}\right) \ll_{n e}\left(\Phi_{i}, \Psi_{i}\right) \ll_{n e} E$ and $E \ll_{n e}\left(\tilde{\Phi}_{i}, \tilde{\Psi}_{i}\right) \ll_{n e}\left(\tilde{\Phi}_{i+1}, \tilde{\Psi}_{i+1}\right)$ and $\left(\tilde{\Phi}_{i}, \tilde{\Psi}_{i}\right)=T\left(\Phi_{i}, \Psi_{i}\right)$. Furthermore, the odd indexed period-two points are saddles and the even indexed period-two points are repellers. The 
global stable manifolds are given by

$$
\begin{aligned}
& \mathcal{W}^{s}\left(\left\{\left(\Phi_{2 k+1}, \Psi_{2 k+1}\right),\left(\tilde{\Phi}_{2 k+1}, \tilde{\Psi}_{2 k+1}\right)\right\}\right)=\mathcal{W}^{s}\left(\Phi_{2 k+1}, \Psi_{2 k+1}\right) \cup \mathcal{W}^{s}\left(\tilde{\Phi}_{2 k+1}, \tilde{\Psi}_{2 k+1}\right), \\
& \quad k=0, \ldots, n,
\end{aligned}
$$

where $\mathcal{W}^{s}\left(\Phi_{2 k+1}, \Psi_{2 k+1}\right)$ and $\mathcal{W}^{s}\left(\tilde{\Phi}_{2 k+1}, \tilde{\Psi}_{2 k+1}\right)$ are the graphs of a continuous strictly increasing function such that $\mathcal{W}^{s}\left(\tilde{\Phi}_{2 k+1}, \tilde{\Psi}_{2 k+1}\right)=T\left(\mathcal{W}^{s}\left(\Phi_{2 k+1}, \Psi_{2 k+1}\right)\right)$ with endpoints at $\left(\tilde{\Phi}_{2 k}, \tilde{\Psi}_{2 k}\right),\left(\tilde{\Phi}_{2 k+2}, \tilde{\Psi}_{2 k+2}\right)$ and $\left(\Phi_{2 k+2}, \Psi_{2 k+2}\right),\left(\Phi_{2 k}, \Psi_{2 k}\right), k=0, \ldots, n-1$, respectively, where $\left(\tilde{\Phi}_{0}, \tilde{\Psi}_{0}\right)=\left(\Phi_{0}, \Psi_{0}\right)=E$. The curve

$$
\mathcal{C}=\bigcup_{k=0}^{n}\left(\mathcal{W}^{s}\left(\Phi_{2 k+1}, \Psi_{2 k+1}\right) \cup \mathcal{W}^{s}\left(\tilde{\Phi}_{2 k+1}, \tilde{\Psi}_{2 k+1}\right) \cup\left\{\left(\Phi_{2 k}, \Psi_{2 k}\right),\left(\tilde{\Phi}_{2 k}, \tilde{\Psi}_{2 k}\right)\right\}\right)
$$

separates $\mathcal{R}$ into two components $\mathcal{W}^{-}$and $\mathcal{W}^{+}$, which are basins of attraction of $(0, \infty)$ and $(\infty, 0)$, respectively, where

$$
\begin{aligned}
& \mathcal{W}^{-}=\left\{(x, y) \mid(x, y) \preceq_{s e}\left(\tilde{x}_{0}, \tilde{y}_{0}\right) \text { for some }\left(\tilde{x}_{0}, \tilde{y}_{0}\right) \in \mathcal{C}\right\} ; \\
& \mathcal{W}^{+}=\left\{(x, y) \mid\left(\tilde{x}_{1}, \tilde{y}_{1}\right) \preceq_{s e}(x, y) \text { for some }\left(\tilde{x}_{1}, \tilde{y}_{1}\right) \in \mathcal{C}\right\} .
\end{aligned}
$$

Further, for $k=0, \ldots, n$, we have

$$
\begin{aligned}
& \mathcal{B}\left(\left\{\left(\Phi_{2 k+1}, \Psi_{2 k+1}\right),\left(\tilde{\Phi}_{2 k+1}, \tilde{\Psi}_{2 k+1}\right)\right\}\right)=\mathcal{W}^{s}\left(\Phi_{2 k+1}, \Psi_{2 k+1}\right) \cup \mathcal{W}^{s}\left(\tilde{\Phi}_{2 k+1}, \tilde{\Psi}_{2 k+1}\right) ; \\
& \mathcal{B}\left(\left\{\left(\Phi_{2 k}, \Psi_{2 k}\right),\left(\tilde{\Phi}_{2 k}, \tilde{\Psi}_{2 k}\right)\right\}\right)=\left\{\left(\Phi_{2 k}, \Psi_{2 k}\right),\left(\tilde{\Phi}_{2 k}, \tilde{\Psi}_{2 k}\right)\right\} .
\end{aligned}
$$

The global unstable manifolds are given by

$$
\begin{aligned}
& \mathcal{W}^{u}\left(\left\{\left(\Phi_{2 k+1}, \Psi_{2 k+1}\right),\left(\tilde{\Phi}_{2 k+1}, \tilde{\Psi}_{2 k+1}\right)\right\}\right)=\mathcal{W}^{u}\left(\Phi_{2 k+1}, \Psi_{2 k+1}\right) \cup \mathcal{W}^{u}\left(\tilde{\Phi}_{2 k+1}, \tilde{\Psi}_{2 k+1}\right), \\
& \quad k=0, \ldots, n,
\end{aligned}
$$

where $\mathcal{W}^{u}\left(\Phi_{2 k+1}, \Psi_{2 k+1}\right)$ and $\mathcal{W}^{u}\left(\tilde{\Phi}_{2 k+1}, \tilde{\Psi}_{2 k+1}\right)$ are the graphs of continuous strictly decreasing functions such that $\mathcal{W}^{u}\left(\tilde{\Phi}_{2 k+1}, \tilde{\Psi}_{2 k+1}\right)=T\left(\mathcal{W}^{u}\left(\Phi_{2 k+1}, \Psi_{2 k+1}\right)\right)$ with endpoints at $(0, \infty)$ and $(\infty, 0)$.

Proof In view of Corollary 2, the set $\operatorname{int}\left(Q_{1}(E)\right) \cup \operatorname{int}\left(Q_{3}(E)\right)$ contains an odd number of minimal period-two solutions $\left\{\left(\Phi_{i}, \Psi_{i}\right),\left(\tilde{\Phi}_{i}, \tilde{\Psi}_{i}\right)\right\}, i=1, \ldots, 2 n+1$, such that $\left(\Phi_{i+1}, \Psi_{i+1}\right) \ll_{n e}$ $\left(\Phi_{i}, \Psi_{i}\right) \ll_{n e} E$ and $E \ll_{n e}\left(\tilde{\Phi}_{i}, \tilde{\Psi}_{i}\right) \ll_{n e}\left(\tilde{\Phi}_{i+1}, \tilde{\Psi}_{i+1}\right)$ and $\left(\tilde{\Phi}_{i}, \tilde{\Psi}_{i}\right)=T\left(\Phi_{i}, \Psi_{i}\right)$. Furthermore, the odd indexed period-two points are saddles and the even indexed period-two points are repellers. The map $T^{2}$ satisfies all conditions of Theorems 6, 7 and 8, which yields the existence of the global stable and unstable manifolds with the above properties. In view of Theorem 7, for $\left(x_{0}, y_{0}\right) \in \mathcal{W}^{-} \cap \mathcal{R}$, there exists $n_{0}>0$ such that

$$
T^{n}\left(x_{0}, y_{0}\right) \in \bigcup_{k=0}^{n}\left(\operatorname{int}\left(Q_{2}\left(\Phi_{2 k+1}, \Psi_{2 k+1}\right)\right) \cup \operatorname{int}\left(Q_{2}\left(\tilde{\Phi}_{2 k+1}, \tilde{\Psi}_{2 k+1}\right)\right)\right) \cap \mathcal{R}, \quad n>n_{0},
$$




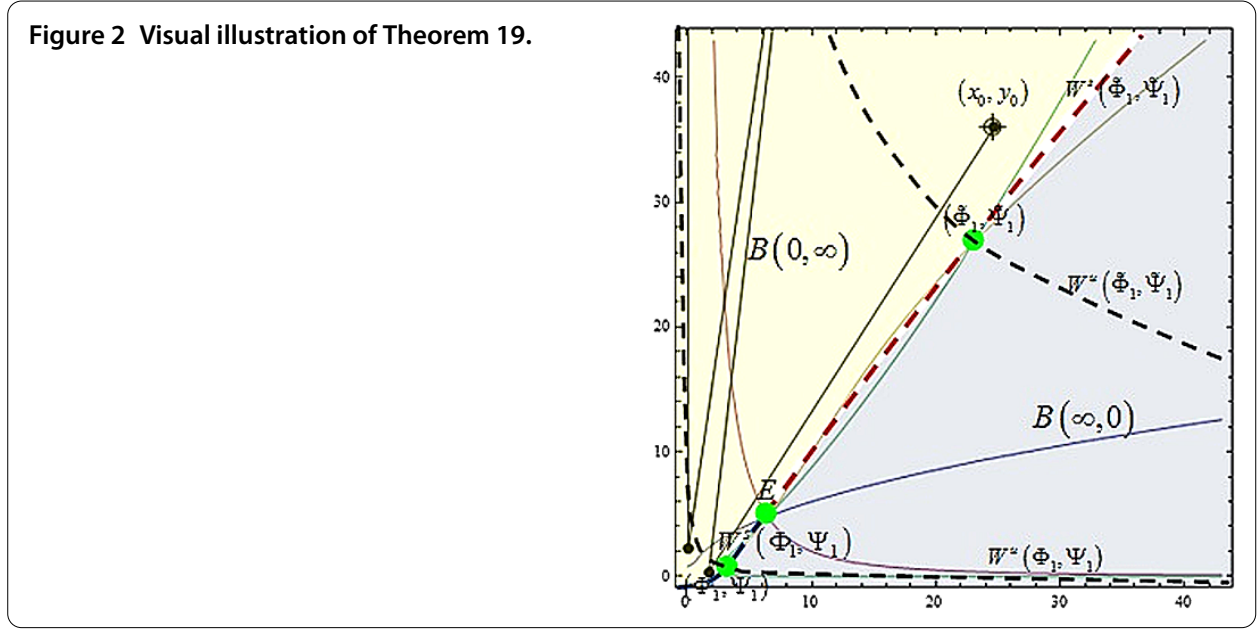

and for $\left(x_{0}, y_{0}\right) \in \mathcal{W}^{+} \cap \mathcal{R}$, there exists $n_{1}>0$ such that

$$
T^{n}\left(x_{0}, y_{0}\right) \in \bigcup_{k=0}^{n}\left(\operatorname{int}\left(Q_{4}\left(\Phi_{2 k+1}, \Psi_{2 k+1}\right)\right) \cup \operatorname{int}\left(Q_{4}\left(\tilde{\Phi}_{2 k+1}, \tilde{\Psi}_{2 k+1}\right)\right)\right) \cap \mathcal{R}, \quad n>n_{1} .
$$

Similarly as in the proof of Theorem 17, one can prove that

$$
\bigcup_{k=0}^{n}\left(\operatorname{int}\left(Q_{2}\left(\Phi_{2 k+1}, \Psi_{2 k+1}\right)\right) \cup \operatorname{int}\left(Q_{2}\left(\tilde{\Phi}_{2 k+1}, \tilde{\Psi}_{2 k+1}\right)\right)\right) \cap \mathcal{R} \subset \mathcal{B}(0, \infty)
$$

and

$$
\bigcup_{k=0}^{n}\left(\operatorname{int}\left(Q_{4}\left(\Phi_{2 k+1}, \Psi_{2 k+1}\right)\right) \cup \operatorname{int}\left(Q_{4}\left(\tilde{\Phi}_{2 k+1}, \tilde{\Psi}_{2 k+1}\right)\right)\right) \cap \mathcal{R} \subset \mathcal{B}(\infty, 0)
$$

which completes the proof.

See Figure 2 for visual illustration of Theorem 19.

\subsection{The case where at least one of the equilibrium points is non-hyperbolic and all minimal period-two solutions are hyperbolic $\left(\Delta_{1}=0\right.$ and $\left.D(p) \neq 0\right)$}

Theorem 20 Assume that $\Delta_{1}=0, \Delta_{2} \neq 0$. Then there exist two equilibrium points $E_{1}\left(\bar{x}_{1}, \bar{y}_{1}\right)$ and $E_{2}\left(\bar{x}_{2}, \bar{y}_{2}\right)$ such that $E_{1} \ll_{n e} E_{2}$, and the following hold:

(a) If $E_{2}$ is non-hyperbolic, then there exist two invariant curves $\mathcal{C}_{1}^{+}$and $\mathcal{C}_{2}^{+}$that are contained in $Q_{1}\left(E_{2}\right)$ with endpoint in $\partial Q_{1}\left(E_{2}\right)$, which are the graphs of continuous strictly increasing functions. Further, there exists the global stable manifold $\mathcal{W}^{s}\left(E_{1}\right)$ with endpoint at $E_{2}$, which is a graph of a continuous increasing function and the global unstable manifold $\mathcal{W}^{u}\left(E_{1}\right)$, which is a graph of a continuous decreasing function. Every solution $\left\{\left(x_{n}, y_{n}\right)\right\}$ which starts below $\mathcal{W}^{s}\left(E_{1}\right) \cup \mathcal{C}_{1}^{+}$in the south-east ordering is asymptotic to $(0, \infty)$, and every solution $\left\{\left(x_{n}, y_{n}\right)\right\}$ which starts above $\mathcal{W}^{s}\left(E_{1}\right) \cup \mathcal{C}_{2}^{+}$in the south-east ordering is asymptotic to $(\infty, 0)$. The first quadrant of initial condition $Q_{1}=\left\{\left(x_{0}, y_{0}\right): x_{0}>0, y_{0} \geq 0\right\}$ is the union of four disjoint basins of attraction, $Q_{1}=\mathcal{B}(0, \infty) \cup \mathcal{B}(\infty, 0) \cup \mathcal{B}\left(E_{1}\right) \cup \mathcal{B}\left(E_{2}\right)$, where 


$$
\begin{aligned}
& \mathcal{B}\left(E_{1}\right)=\mathcal{W}^{s}\left(E_{1}\right) \text { and } \\
& \mathcal{B}\left(E_{2}\right)=\left\{(x, y) \in \mathcal{R}:\left(\tilde{x}_{1}, \tilde{y}_{1}\right) \preceq_{s e}(x, y) \preceq_{s e}\left(\tilde{x}_{2}, \tilde{y}_{2}\right)\right. \\
&\text { for some } \left.\left(\tilde{x}_{1}, \tilde{y}_{1}\right) \in \mathcal{C}_{1}^{+} \text {and }\left(\tilde{x}_{2}, \tilde{y}_{2}\right) \in \mathcal{C}_{2}^{+}\right\}, \\
& \mathcal{B}(0, \infty)=\left\{(x, y) \mid(x, y) \preceq_{s e}\left(\tilde{x}_{0}, \tilde{y}_{0}\right) \text { for some }\left(\tilde{x}_{0}, \tilde{y}_{0}\right) \in \mathcal{W}^{s}\left(E_{1}\right) \cup \mathcal{C}_{1}^{+}\right\}, \\
& \mathcal{B}(\infty, 0)=\left\{(x, y) \mid\left(\tilde{x}_{1}, \tilde{y}_{1}\right) \preceq_{s e}(x, y) \text { for some }\left(\tilde{x}_{1}, \tilde{y}_{1}\right) \in \mathcal{W}^{s}\left(E_{1}\right) \cup \mathcal{C}_{2}^{+}\right\} .
\end{aligned}
$$

(b) If $E_{1}$ is non-hyperbolic, then there exist two invariant curves $\mathcal{C}_{1}^{-}$and $\mathcal{C}_{2}^{-}$that are contained in $Q_{3}\left(E_{1}\right)$ with endpoint in $\partial Q_{3}\left(E_{1}\right)$, which are the graphs of continuous strictly increasing functions. Further, there exists the global stable manifold $\mathcal{W}^{s}\left(E_{2}\right)$ with endpoint at $E_{1}$, which is a graph of a continuous increasing function, and the global unstable manifold $\mathcal{W}^{u}\left(E_{2}\right)$, which is a graph of a continuous decreasing function. Every solution $\left\{\left(x_{n}, y_{n}\right)\right\}$ which starts below $\mathcal{W}^{s}\left(E_{2}\right) \cup \mathcal{C}_{1}^{-}$in the south-east ordering is asymptotic to $(0, \infty)$, and every solution $\left\{\left(x_{n}, y_{n}\right)\right\}$ which starts above $\mathcal{W}^{s}\left(E_{2}\right) \cup \mathcal{C}_{2}^{-}$in the south-east ordering is asymptotic to $(\infty, 0)$. The first quadrant of initial condition $Q_{1}=\left\{\left(x_{0}, y_{0}\right): x_{0}>0, y_{0} \geq 0\right\}$ is the union of four disjoint basins of attraction, $Q_{1}=\mathcal{B}(0, \infty) \cup \mathcal{B}(\infty, 0) \cup \mathcal{B}\left(E_{1}\right) \cup \mathcal{B}\left(E_{2}\right)$, where $\mathcal{B}\left(E_{2}\right)=\mathcal{W}^{s}\left(E_{2}\right)$ and

$$
\begin{aligned}
& \mathcal{B}\left(E_{1}\right)=\left\{(x, y) \in \mathcal{R}:\left(\tilde{x}_{1}, \tilde{y}_{1}\right) \preceq_{s e}(x, y) \preceq_{s e}\left(\tilde{x}_{2}, \tilde{y}_{2}\right)\right. \\
&\text { for some } \left.\left(\tilde{x}_{1}, \tilde{y}_{1}\right) \in \mathcal{C}_{1}^{-} \text {and }\left(\tilde{x}_{2}, \tilde{y}_{2}\right) \in \mathcal{C}_{2}^{-}\right\}, \\
& \mathcal{B}(0, \infty)=\left\{(x, y) \mid(x, y) \preceq_{s e}\left(\tilde{x}_{0}, \tilde{y}_{0}\right) \text { for some }\left(\tilde{x}_{0}, \tilde{y}_{0}\right) \in \mathcal{W}^{s}\left(E_{2}\right) \cup \mathcal{C}_{1}^{-}\right\}, \\
& \mathcal{B}(\infty, 0)=\left\{(x, y) \mid\left(\tilde{x}_{1}, \tilde{y}_{1}\right) \preceq_{s e}(x, y) \text { for some }\left(\tilde{x}_{1}, \tilde{y}_{1}\right) \in \mathcal{W}^{s}\left(E_{2}\right) \cup \mathcal{C}_{2}^{-}\right\} .
\end{aligned}
$$

Proof We will prove statement (a). The proof of statement (b) is similar. By Theorem 12 we have that $T$ has two equilibrium points $E_{1}$ and $E_{2}$ such that $E_{1} \ll_{n e} E_{2}$. Then either $E_{1}$ is non-hyperbolic and $E_{2}$ is a saddle point or $E_{2}$ is non-hyperbolic and $E_{1}$ is a saddle point. Assume that $E_{2}$ is non-hyperbolic. From Descartes' rule of signs applied to (8) we have that $a_{2}<A_{1} c_{2}$. Lemma 2 implies that $T^{n}\left(x_{0}, y_{0}\right)$ is asymptotic to either $(0, \infty)$ or $(\infty, 0)$ or to an equilibrium point for all $\left(x_{0}, y_{0}\right) \in \mathcal{R}$. Let $\mathcal{B}(0, \infty)$ be the basin of attraction of $(0, \infty)$, and let $\mathcal{B}(\infty, 0)$ be the basin of attraction of $(\infty, 0)$. By using Theorem 9 one can prove that $\operatorname{int}\left(Q_{2}\left(E_{2}\right)\right) \subset \mathcal{B}(0, \infty)$ and $\operatorname{int}\left(Q_{4}\left(E_{2}\right)\right) \subset \mathcal{B}(\infty, 0)$. Let $\mathcal{C}_{1}^{+}$denote the boundary of $\mathcal{B}(0, \infty)$ considered as a subset of $Q_{1}\left(E_{2}\right)$ and $\mathcal{C}_{2}^{+}$denote the boundary of $\mathcal{B}(\infty, 0)$ considered as a subset of $Q_{1}\left(E_{2}\right)$. It is easy to see that $E \in \mathcal{C}_{1}^{+}$and $E \in \mathcal{C}_{2}^{+}$. Now Theorems 6,7 and 8 yield the existence of the global stable manifold $\mathcal{W}^{s}\left(E_{1}\right)$ and the global unstable manifold $\mathcal{W}^{u}\left(E_{1}\right)$, where $\mathcal{W}^{s}\left(E_{1}\right)$ has an endpoint at $E_{2}$ and it is a graph of an increasing function. Similarly as in the proof of Theorem 17, one can see that

$$
\begin{aligned}
& \mathcal{W}^{-}=\left\{(x, y) \mid(x, y) \preceq_{s e}\left(\tilde{x}_{0}, \tilde{y}_{0}\right) \text { for some }\left(\tilde{x}_{0}, \tilde{y}_{0}\right) \in \mathcal{W}^{s}\left(E_{1}\right)\right\} \subset \mathcal{B}(0, \infty), \\
& \mathcal{W}^{+}=\left\{(x, y) \mid\left(\tilde{x}_{1}, \tilde{y}_{1}\right) \preceq_{s e}(x, y) \text { for some }\left(\tilde{x}_{1}, \tilde{y}_{1}\right) \in \mathcal{W}^{s}\left(E_{1}\right)\right\} \subset \mathcal{B}(\infty, 0),
\end{aligned}
$$

and $\mathcal{B}\left(E_{1}\right)=\mathcal{W}^{s}\left(E_{1}\right)$.

Similarly as in Claim 1, one can prove the following claim. 
Claim 2 Let $\mathcal{C}_{1}^{+}$and $\mathcal{C}_{1}^{+}$be the sets defined as above. Then we have the following:

(a) If $\left(x_{0}, y_{0}\right) \in \mathcal{B}(\infty, 0)$, then $\left(x_{1}, y_{1}\right) \in \mathcal{B}(\infty, 0)$ for all $\left(x_{0}, y_{0}\right) \preceq_{s e}\left(x_{1}, y_{1}\right)$;

(b) If $\left(x_{0}, y_{0}\right) \in \mathcal{B}(0, \infty)$, then $\left(x_{1}, y_{1}\right) \in \mathcal{B}(0, \infty)$ for all $\left(x_{1}, y_{1}\right) \preceq_{s e}\left(x_{0}, y_{0}\right)$;

(c) If $\left(x_{0}, y_{0}\right) \in \mathcal{C}_{1}^{+}$, then $\left(x_{1}, y_{1}\right) \in \operatorname{int}(\mathcal{B}(0, \infty))$ for all $\left(x_{1}, y_{1}\right) \ll_{s e}\left(x_{0}, y_{0}\right)$ and $\left(x_{1}, y_{1}\right) \notin \overline{\mathcal{B}(0, \infty)}$ for all $\left(x_{0}, y_{0}\right) \ll_{\text {se }}\left(x_{1}, y_{1}\right)$;

(d) If $\left(x_{0}, y_{0}\right) \in \mathcal{C}_{2}^{+}$, then $\left(x_{1}, y_{1}\right) \in \operatorname{int}(\mathcal{B}(\infty, 0))$ for all $\left(x_{0}, y_{0}\right) \ll_{s e}\left(x_{1}, y_{1}\right)$ and $\left(x_{1}, y_{1}\right) \notin \overline{\mathcal{B}(\infty, 0)}$ for all $\left(x_{1}, y_{1}\right) \ll_{\text {se }}\left(x_{0}, y_{0}\right) ;$

(e) $\mathcal{C}_{1}^{+} \cap \operatorname{int}\left(Q_{1}\left(E_{2}\right)\right) \neq 0$ and $\mathcal{C}_{2}^{+} \cap \operatorname{int}\left(Q_{1}\left(E_{2}\right)\right) \neq 0$;

(f) $T\left(\mathcal{C}_{1}^{+}\right) \subseteq \mathcal{C}_{1}^{+}$and $T\left(\mathcal{C}_{2}^{+}\right) \subseteq \mathcal{C}_{2}^{+}$;

(g) $\left(x_{0}, y_{0}\right),\left(x_{1}, y_{1}\right) \in \mathcal{C}_{1}^{+} \Rightarrow\left(x_{0}, y_{0}\right) \ll_{n e}\left(x_{1}, y_{1}\right)$ or $\left(x_{1}, y_{1}\right) \ll_{n e}\left(x_{0}, y_{0}\right)$;

(h) $\left(x_{0}, y_{0}\right),\left(x_{1}, y_{1}\right) \in \mathcal{C}_{2}^{+} \Rightarrow\left(x_{0}, y_{0}\right) \ll_{n e}\left(x_{1}, y_{1}\right)$ or $\left(x_{1}, y_{1}\right) \ll_{n e}\left(x_{0}, y_{0}\right)$.

Proof We prove statement (f). Since $\mathcal{W}^{s}\left(E_{1}\right) \cup \mathcal{C}_{1}^{+}$is the boundary of $\mathcal{B}(0, \infty)$ similar as in the proof of statement (d) of Claim 1, we have that $T\left(\mathcal{W}^{s}\left(E_{1}\right) \cup \mathcal{C}_{1}^{+}\right) \subseteq \mathcal{W}^{s}\left(E_{1}\right) \cup \mathcal{C}_{1}^{+}$. Take $\left(x_{0}, y_{0}\right) \in \mathcal{C}_{1}^{+} \backslash E_{2}$. If $T\left(x_{0}, y_{0}\right) \in \mathcal{W}^{s}\left(E_{1}\right)$, then $T^{n}\left(x_{0}, y_{0}\right) \rightarrow E_{1}$ as $n \rightarrow \infty$, which is in contradiction with $\mathcal{B}\left(E_{1}\right)=\mathcal{W}^{s}\left(E_{1}\right)$ and $\mathcal{B}\left(E_{1}\right) \cap \mathcal{C}_{1}^{+}=\left\{E_{2}\right\}$. Hence, $T\left(x_{0}, y_{0}\right) \in \mathcal{C}_{1}^{+}$which implies that $T\left(\mathcal{C}_{1}^{+}\right) \subseteq \mathcal{C}_{1}^{+}$. The corresponding assertion for $\mathcal{C}_{2}^{+}$is proved in a similar fashion.

Now, we prove that $\mathcal{C}_{1}^{+}$and $\mathcal{C}_{2}^{+}$are the graphs of continuous strictly increasing functions. Let $J$ be the projection of $\mathcal{C}_{1}^{+}$onto the first coordinate. From (g), no two distinct elements of $\mathcal{C}_{1}^{+}$can be weakly related nor can they have the same projection onto the first coordinate. Further, $J$ contains $\left[x_{1}, x_{2}\right]$ whenever $x_{1} \leq x_{2}$ belong to $J$. Indeed, if $x_{1} \leq x_{2}$ belong to $J$, then there exist $y_{1}$ and $y_{2}$ such that $\left(x_{1}, y_{1}\right),\left(x_{2}, y_{2}\right) \in \mathcal{C}_{1}^{+}$. From (c), we have that $\operatorname{int}\left(Q_{2}\left(x_{2}, y_{2}\right)\right) \subset$ $\operatorname{int}(\mathcal{B}(0, \infty))$ and $\operatorname{int}\left(Q_{4}\left(x_{1}, y_{1}\right)\right) \subset \overline{\operatorname{int}(\mathcal{B}(\infty, 0))}$. By using this it is easy to see that $(x, \hat{y}) \in \mathcal{C}_{1}^{+}$ if and only if $\hat{y}=\inf \left\{y: y \in\left[y_{1}, y_{2}\right]\right.$ and $\left.(x, y) \in \operatorname{int}(\mathcal{B}(0, \infty))\right\}$, where $x \in\left[x_{1}, x_{2}\right]$. This implies that $J$ contains $\left[x_{1}, x_{2}\right]$. By $(\mathrm{e}), \mathcal{C}_{1}^{+} \cap \operatorname{int}\left(Q_{1}\left(E_{2}\right)\right) \neq 0$. From this it follows that $J$ is an interval such that $\operatorname{int}(J) \neq \emptyset$ and $\mathcal{C}_{1}^{+}$is a connected set. Since points on $\mathcal{C}_{1}^{+}$are non-comparable, $\mathcal{C}_{1}^{+}$ is the graph of a strictly increasing function $f(x)$ of $x \in J$. If there is a jump discontinuity at $x_{0} \in J$, let $y_{-}$and $y_{+}$respectively be the left and right (distinct) limits of $f(x)$ as $x$ approaches $x_{0}$, respectively. The points $\left(x_{0}, y_{-}\right)$and $\left(x_{0}, y_{+}\right)$are comparable in $\preceq_{s e}$-ordering, which is in contradiction with (g). Thus $f(x)$ is a continuous function. Since $T$ has no equilibrium points in $Q_{1}\left(E_{2}\right)$, similarly as in the proof of Theorem 1 [3], one can prove that $\mathcal{C}_{1}^{+}$has its endpoints in $\partial Q_{1}\left(E_{2}\right)$. The proof that considers $\mathcal{C}_{2}^{+}$is similar and will be skipped.

Take $\left(x_{1}, y_{1}\right) \in \mathcal{C}_{1}^{+}$and $\left(x_{2}, y_{2}\right) \in \mathcal{C}_{2}^{+}$. Since $E_{2}$ is the only equilibrium point in $Q_{1}\left(E_{2}\right)$ and $(0, \infty),(\infty, 0) \notin Q_{1}\left(E_{2}\right)$ we have that $T^{n}\left(x_{1}, y_{1}\right)$ and $T^{n}\left(x_{2}, y_{2}\right)$ converge to $E_{2}$ as $n \rightarrow \infty$. If $\left(\tilde{x}_{1}, \tilde{y}_{1}\right) \preceq_{s e}(x, y) \preceq_{s e}\left(\tilde{x}_{2}, \tilde{y}_{2}\right)$ for some $\left(\tilde{x}_{1}, \tilde{y}_{1}\right) \in \mathcal{C}_{1}^{+}$and $\left(\tilde{x}_{2}, \tilde{y}_{2}\right) \in \mathcal{C}_{2}^{+}$, since $T$ is competitive, we get $T^{n}\left(\tilde{x}_{1}, \tilde{y}_{1}\right) \preceq_{s e} T^{n}(x, y) \preceq_{s e} T^{n}\left(\tilde{x}_{2}, \tilde{y}_{2}\right)$, which implies $T^{n}(x, y) \rightarrow E_{2}$ as $n \rightarrow \infty$.

Based on a series of numerical simulations, we propose the following conjecture.

See Figure 3 for visual illustration of Theorem 20.

Conjecture 2 Suppose that all assumptions of Theorem 20 are satisfied, then the following hold:

(a) $\mathcal{B}\left(E_{2}\right)=\mathcal{C}_{1}^{+}=\mathcal{C}_{2}^{+}$;

(b) $\mathcal{B}\left(E_{1}\right)=\mathcal{C}_{1}^{-}=\mathcal{C}_{2}^{-}$.

Theorem 21 Assume that $\Delta_{1}=0, \Delta_{2}=0$ and $a_{2}<A_{1} c_{2}$. Then there exist the unique nonhyperbolic equilibrium point $E(\bar{x}, \bar{y})$ and two invariant curves $\mathcal{C}^{-}$and $\mathcal{C}^{+}$with endpoint in 

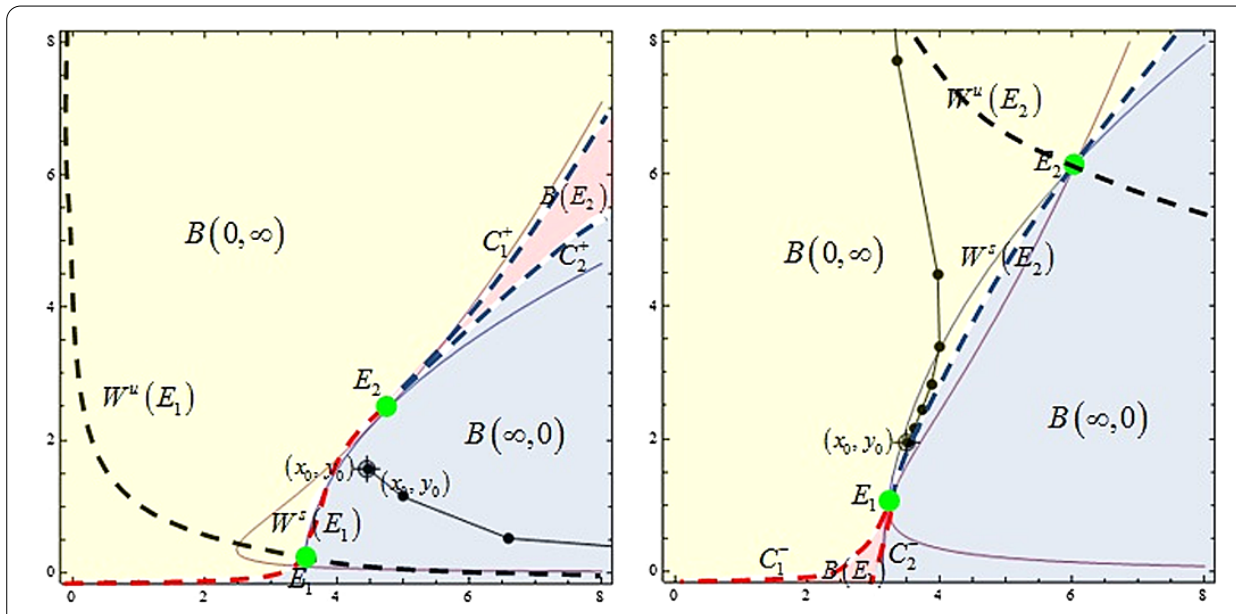

Figure 3 Visual illustration of statements (a) and (b) of Theorem 20.

Figure 4 Visual illustration of Theorem 21.

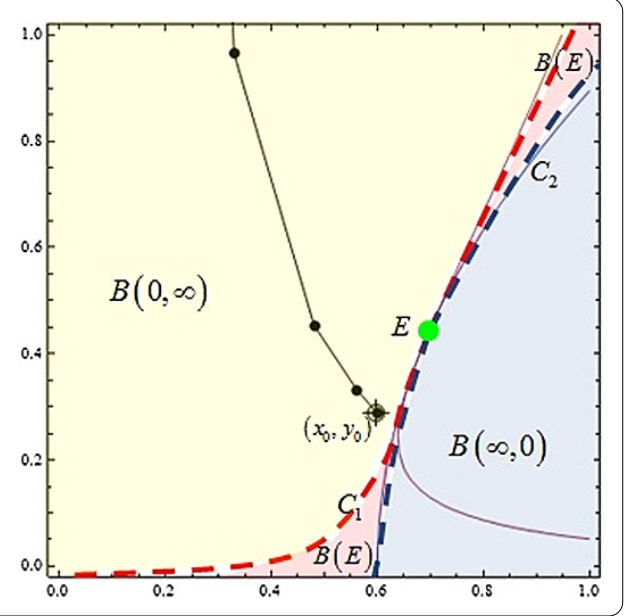

$\partial R$, which are the graphs of continuous strictly increasing functions. Every solution $\left\{\left(x_{n}, y_{n}\right)\right\}$ which starts below $\mathcal{C}_{1}$ in the south-east ordering is asymptotic to $(0, \infty)$ and every solution $\left\{\left(x_{n}, y_{n}\right)\right\}$ which starts above $\mathcal{C}_{2}$ in the south-east ordering is asymptotic to $(\infty, 0)$. The first quadrant of initial condition $Q_{1}=\left\{\left(x_{0}, y_{0}\right): x_{0}>0, y_{0} \geq 0\right\}$ is the union of three disjoint basins of attraction, $Q_{1}=\mathcal{B}(0, \infty) \cup \mathcal{B}(\infty, 0) \cup \mathcal{B}(E)$, where

$$
\begin{aligned}
& \mathcal{B}(E)=\left\{(x, y) \in \mathcal{R}:\left(\tilde{x}_{1}, \tilde{y}_{1}\right) \preceq_{s e}(x, y) \preceq_{s e}\left(\tilde{x}_{2}, \tilde{y}_{2}\right) \text { for some }\left(\tilde{x}_{1}, \tilde{y}_{1}\right) \in \mathcal{C}_{1} \text { and }\left(\tilde{x}_{2}, \tilde{y}_{2}\right) \in \mathcal{C}_{2}\right\} ; \\
& \mathcal{B}(0, \infty)=\left\{(x, y) \mid(x, y) \preceq_{s e}\left(\tilde{x}_{0}, \tilde{y}_{0}\right) \text { for some }\left(\tilde{x}_{0}, \tilde{y}_{0}\right) \in \mathcal{C}_{1}\right\} ; \\
& \mathcal{B}(\infty, 0)=\left\{(x, y) \mid\left(\tilde{x}_{1}, \tilde{y}_{1}\right) \preceq_{s e}(x, y) \text { for some }\left(\tilde{x}_{1}, \tilde{y}_{1}\right) \in \mathcal{C}_{2}\right\} .
\end{aligned}
$$

Proof The proof is similar to the proof of Theorem 20 and will be skipped.

Based on a series of numerical simulations, we propose the following conjecture.

See Figure 4 for visual illustration of Theorem 21. 
Conjecture 3 Suppose that all assumptions of Theorem 21 are satisfied, then the following holds:

$$
\mathcal{B}(E)=\mathcal{C}_{1}=\mathcal{C}_{2}
$$

Appendix: Values of coefficients $p_{i}$ for $i=0, \ldots, 22$

$$
\begin{aligned}
& p_{0}=a_{2}^{2} b_{1}^{4}\left(c_{2}^{4} A_{1}^{12}-4 a_{2} c_{2}^{3} A_{1}^{11}+6 a_{2}^{2} c_{2}^{2} A_{1}^{10}+a_{2}^{4} A_{1}^{8}+a_{2}^{3}\left(b_{1}^{12} c_{2}^{7}-4 A_{1}^{9} c_{2}\right)\right) \text {, } \\
& p_{1}=a_{2} A_{1}^{2} b_{1}^{2}\left(c_{2}^{4} A_{1}^{12}+a_{2} c_{2}^{3}\left(3 b_{1}^{4} c_{2}^{2}-4 A_{1}^{3}\right) A_{1}^{8}+2 a_{2}^{2} c_{2}^{2}\left(3 A_{1}^{3}-8 b_{1}^{4} c_{2}^{2}\right) A_{1}^{7}+7 a_{2}^{5} b_{1}^{4} c_{2} A_{1}^{4}\right. \\
& \left.+a_{2}^{4}\left(A_{1}^{8}-24 A_{1}^{5} b_{1}^{4} c_{2}^{2}\right)+a_{2}^{3}\left(c_{2}^{7} b_{1}^{12}+30 A_{1}^{6} c_{2}^{3} b_{1}^{4}-4 A_{1}^{9} c_{2}\right)\right) \text {, } \\
& p_{2}=c_{2}^{4} A_{1}^{16}+2 a_{2} c_{2}^{3}\left(b_{1}^{4} c_{2}^{2}-2 A_{1}^{3}\right) A_{1}^{12}+a_{2}^{3} c_{2}\left(c_{2}^{6} b_{1}^{12}-8 A_{1}^{3} c_{2}^{4} b_{1}^{8}-8 A_{1}^{6} c_{2}^{2} b_{1}^{4}-4 A_{1}^{9}\right) A_{1}^{4} \\
& +2 a_{2}^{6}\left(7 A_{1}^{4} c_{2}^{2} b_{1}^{8}+4 A_{1}^{7} b_{1}^{4}\right)-2 a_{2}^{5}\left(13 b_{1}^{4} c_{2} A_{1}^{8}+18 b_{1}^{8} c_{2}^{3} A_{1}^{5}\right)+a_{2}^{2}\left(6 A_{1}^{14} c_{2}^{2}-4 A_{1}^{11} b_{1}^{4} c_{2}^{4}\right) \\
& +a_{2}^{4}\left(5 c_{2}^{8} b_{1}^{16}+30 A_{1}^{6} c_{2}^{4} b_{1}^{8}+28 A_{1}^{9} c_{2}^{2} b_{1}^{4}+A_{1}^{12}\right), \\
& p_{3}=A_{1} b_{1}^{2}\left(c_{2}^{5} A_{1}^{13}+a_{2} c_{2}^{4}\left(3 c_{2}^{2} b_{1}^{4}+2 A_{1}^{3}\right) A_{1}^{9}+a_{2}^{2} c_{2}^{3}\left(c_{2}^{4} b_{1}^{8}-6 A_{1}^{3} c_{2}^{2} b_{1}^{4}-22 A_{1}^{6}\right) A_{1}^{5}\right. \\
& +4 a_{2}^{3} c_{2}^{2}\left(c_{2}^{6} b_{1}^{12}-9 A_{1}^{6} c_{2}^{2} b_{1}^{4}+11 A_{1}^{9}\right) A_{1}+7 a_{2}^{6}\left(A_{1} c_{2}^{3} b_{1}^{8}+6 A_{1}^{4} c_{2} b_{1}^{4}\right) \\
& \left.+a_{2}^{5}\left(10 A_{1}^{8}-123 b_{1}^{4} c_{2}^{2} A_{1}^{5}-8 b_{1}^{8} c_{2}^{4} A_{1}^{2}\right)+a_{2}^{4}\left(2 c_{2}^{7} b_{1}^{12}+120 A_{1}^{6} c_{2}^{3} b_{1}^{4}-35 A_{1}^{9} c_{2}\right)\right), \\
& p_{4}=c_{2}^{4}\left(c_{2}^{2} b_{1}^{4}+8 A_{1}^{3}\right) A_{1}^{12}+a_{2} c_{2}^{3}\left(c_{2}^{4} b_{1}^{8}+4 A_{1}^{3} c_{2}^{2} b_{1}^{4}-32 A_{1}^{6}\right) A_{1}^{8} \\
& +a_{2}^{4}\left(4 c_{2}^{4} b_{1}^{8}+23 A_{1}^{3} c_{2}^{2} b_{1}^{4}+8 A_{1}^{6}\right) A_{1}^{5} \\
& +a_{2}^{2} c_{2}^{2}\left(3 c_{2}^{6} b_{1}^{12}-24 A_{1}^{3} c_{2}^{4} b_{1}^{8}-32 A_{1}^{6} c_{2}^{2} b_{1}^{4}+48 A_{1}^{9}\right) A_{1}^{4}+28 a_{2}^{6} b_{1}^{4}\left(2 c_{2}^{2} b_{1}^{4}+A_{1}^{3}\right) A_{1}^{3} \\
& -a_{2}^{5}\left(95 A_{1}^{4} c_{2}^{3} b_{1}^{8}+64 A_{1}^{7} c_{2} b_{1}^{4}\right) \\
& +a_{2}^{3}\left(10 c_{2}^{9} b_{1}^{16}+4 A_{1}^{3} c_{2}^{7} b_{1}^{12}+58 A_{1}^{6} c_{2}^{5} b_{1}^{8}+40 A_{1}^{9} c_{2}^{3} b_{1}^{4}-32 A_{1}^{12} c_{2}\right), \\
& p_{5}=b_{1}^{2}\left(c_{2}^{5}\left(c_{2}^{2} b_{1}^{4}+6 A_{1}^{3}\right) A_{1}^{10}+a_{2} c_{2}^{4}\left(2 c_{2}^{4} b_{1}^{8}+2 A_{1}^{3} c_{2}^{2} b_{1}^{4}-17 A_{1}^{6}\right) A_{1}^{6}\right. \\
& +3 a_{2}^{2} c_{2}^{3}\left(2 c_{2}^{6} b_{1}^{12}+2 A_{1}^{3} c_{2}^{4} b_{1}^{8}-13 A_{1}^{6} c_{2}^{2} b_{1}^{4}-8 A_{1}^{9}\right) A_{1}^{2} \\
& +2 a_{2}^{3} c_{2}^{2}\left(4 c_{2}^{6} b_{1}^{12}+20 A_{1}^{6} c_{2}^{2} b_{1}^{4}+57 A_{1}^{9}\right) A_{1}+7 a_{2}^{6}\left(2 A_{1} c_{2}^{3} b_{1}^{8}+15 A_{1}^{4} c_{2} b_{1}^{4}\right) \\
& +a_{2}^{5}\left(39 A_{1}^{8}-234 b_{1}^{4} c_{2}^{2} A_{1}^{5}+19 b_{1}^{8} c_{2}^{4} A_{1}^{2}\right) \\
& \left.+a_{2}^{4}\left(c_{2}^{7} b_{1}^{12}-40 A_{1}^{3} c_{2}^{5} b_{1}^{8}+125 A_{1}^{6} c_{2}^{3} b_{1}^{4}-118 A_{1}^{9} c_{2}\right)\right), \\
& p_{6}=c_{2}^{4}\left(c_{2}^{4} b_{1}^{8}+4 A_{1}^{3} c_{2}^{2} b_{1}^{4}+28 A_{1}^{6}\right) A_{1}^{8}+2 a_{2}^{4}\left(-107 c_{2}^{4} b_{1}^{8}-52 A_{1}^{3} c_{2}^{2} b_{1}^{4}+14 A_{1}^{6}\right) A_{1}^{4} \\
& -a_{2} c_{2}^{3}\left(-3 c_{2}^{6} b_{1}^{12}+16 A_{1}^{3} c_{2}^{4} b_{1}^{8}+16 A_{1}^{6} c_{2}^{2} b_{1}^{4}+112 A_{1}^{9}\right) A_{1}^{4} \\
& +2 a_{2}^{3} c_{2}\left(3 c_{2}^{6} b_{1}^{12}+86 A_{1}^{3} c_{2}^{4} b_{1}^{8}+74 A_{1}^{6} c_{2}^{2} b_{1}^{4}-56 A_{1}^{9}\right) A_{1}^{2}+a_{2}^{6}\left(84 A_{1}^{2} c_{2}^{2} b_{1}^{8}+52 A_{1}^{5} b_{1}^{4}\right) \\
& -4 a_{2}^{5}\left(5 A_{1}^{3} c_{2}^{3} b_{1}^{8}+13 A_{1}^{6} c_{2} b_{1}^{4}\right) \\
& +2 a_{2}^{2} c_{2}^{2}\left(5 c_{2}^{8} b_{1}^{16}+6 A_{1}^{3} c_{2}^{6} b_{1}^{12}-3 A_{1}^{6} c_{2}^{4} b_{1}^{8}-16 A_{1}^{9} c_{2}^{2} b_{1}^{4}+84 A_{1}^{12}\right), \\
& p_{7}=b_{1}^{2}\left(-A_{1}^{2} b_{1}^{4} a_{2}^{7}+7\left(c_{2}^{3} b_{1}^{8}+20 A_{1}^{3} c_{2} b_{1}^{4}\right) a_{2}^{6}+\left(62 A_{1} c_{2}^{4} b_{1}^{8}-165 A_{1}^{4} c_{2}^{2} b_{1}^{4}+80 A_{1}^{7}\right) a_{2}^{5}\right. \\
& \text { - }\left(197 c_{2} A_{1}^{8}+90 b_{1}^{4} c_{2}^{3} A_{1}^{5}+10 b_{1}^{8} c_{2}^{5} A_{1}^{2}\right) a_{2}^{4} \\
& +\left(4 c_{2}^{8} b_{1}^{12}-80 A_{1}^{3} c_{2}^{6} b_{1}^{8}+135 A_{1}^{6} c_{2}^{4} b_{1}^{4}+100 A_{1}^{9} c_{2}^{2}\right) a_{2}^{3}
\end{aligned}
$$




$$
\begin{aligned}
& +A_{1} c_{2}^{3}\left(12 c_{2}^{6} b_{1}^{12}+15 A_{1}^{3} c_{2}^{4} b_{1}^{8}+82 A_{1}^{9}\right) a_{2}^{2} \\
& +A_{1}^{2} c_{2}^{4}\left(4 c_{2}^{6} b_{1}^{12}+12 A_{1}^{3} c_{2}^{4} b_{1}^{8}-21 A_{1}^{6} c_{2}^{2} b_{1}^{4}-76 A_{1}^{9}\right) a_{2} \\
& \left.+A_{1}^{6} c_{2}^{5}\left(c_{2}^{4} b_{1}^{8}+2 A_{1}^{3} c_{2}^{2} b_{1}^{4}+11 A_{1}^{6}\right)\right) \text {, } \\
& p_{8}=\left(56 A_{1} c_{2}^{2} b_{1}^{8}+53 A_{1}^{4} b_{1}^{4}\right) a_{2}^{6}+2\left(75 A_{1}^{2} c_{2}^{3} b_{1}^{8}+26 A_{1}^{5} c_{2} b_{1}^{4}\right) a_{2}^{5} \\
& +4\left(14 A_{1}^{9}-65 b_{1}^{4} c_{2}^{2} A_{1}^{6}-74 b_{1}^{8} c_{2}^{4} A_{1}^{3}\right) a_{2}^{4} \\
& +4 A_{1} c_{2}\left(c_{2}^{6} b_{1}^{12}+A_{1}^{3} c_{2}^{4} b_{1}^{8}+26 A_{1}^{6} c_{2}^{2} b_{1}^{4}-56 A_{1}^{9}\right) a_{2}^{3} \\
& +A_{1}^{2} c_{2}^{2}\left(18 c_{2}^{6} b_{1}^{12}+132 A_{1}^{3} c_{2}^{4} b_{1}^{8}+101 A_{1}^{6} c_{2}^{2} b_{1}^{4}+336 A_{1}^{9}\right) a_{2}^{2} \\
& +c_{2}^{3}\left(5 c_{2}^{8} b_{1}^{16}+12 A_{1}^{3} c_{2}^{6} b_{1}^{12}-38 A_{1}^{6} c_{2}^{4} b_{1}^{8}-52 A_{1}^{9} c_{2}^{2} b_{1}^{4}-224 A_{1}^{12}\right) a_{2} \\
& +A_{1}^{4} c_{2}^{4}\left(c_{2}^{6} b_{1}^{12}+2 A_{1}^{6} c_{2}^{2} b_{1}^{4}+56 A_{1}^{9}\right) \text {, } \\
& p_{9}=b_{1}^{2}\left(-2 A_{1} b_{1}^{4} a_{2}^{7}+98 A_{1}^{2} b_{1}^{4} c_{2} a_{2}^{6}+5\left(7 c_{2}^{4} b_{1}^{8}+12 A_{1}^{3} c_{2}^{2} b_{1}^{4}+19 A_{1}^{6}\right) a_{2}^{5}\right. \\
& -5\left(-20 A_{1} c_{2}^{5} b_{1}^{8}+45 A_{1}^{4} c_{2}^{3} b_{1}^{4}+32 A_{1}^{7} c_{2}\right) a_{2}^{4} \\
& -2\left(43 c_{2}^{2} A_{1}^{8}+15 b_{1}^{4} c_{2}^{4} A_{1}^{5}+45 b_{1}^{8} c_{2}^{6} A_{1}^{2}\right) a_{2}^{3} \\
& +2\left(3 c_{2}^{9} b_{1}^{12}-30 A_{1}^{3} c_{2}^{7} b_{1}^{8}+63 A_{1}^{6} c_{2}^{5} b_{1}^{4}+134 A_{1}^{9} c_{2}^{3}\right) a_{2}^{2} \\
& +A_{1} c_{2}^{4}\left(8 c_{2}^{6} b_{1}^{12}+30 A_{1}^{3} c_{2}^{4} b_{1}^{8}-24 A_{1}^{6} c_{2}^{2} b_{1}^{4}-113 A_{1}^{9}\right) a_{2} \\
& \left.+A_{1}^{2} c_{2}^{5}\left(c_{2}^{6} b_{1}^{12}+6 A_{1}^{3} c_{2}^{4} b_{1}^{8}-3 A_{1}^{6} c_{2}^{2} b_{1}^{4}-4 A_{1}^{9}\right)\right) \text {, } \\
& p_{10}=14\left(c_{2}^{2} b_{1}^{8}+2 A_{1}^{3} b_{1}^{4}\right) a_{2}^{6}+2\left(80 A_{1} c_{2}^{3} b_{1}^{8}+67 A_{1}^{4} c_{2} b_{1}^{4}\right) a_{2}^{5} \\
& +2\left(35 A_{1}^{8}-92 b_{1}^{4} c_{2}^{2} A_{1}^{5}-7 b_{1}^{8} c_{2}^{4} A_{1}^{2}\right) a_{2}^{4} \\
& +\left(c_{2}^{7} b_{1}^{12}-264 A_{1}^{3} c_{2}^{5} b_{1}^{8}-184 A_{1}^{6} c_{2}^{3} b_{1}^{4}-280 A_{1}^{9} c_{2}\right) a_{2}^{3} \\
& +4 A_{1} c_{2}^{2}\left(3 c_{2}^{6} b_{1}^{12}+34 A_{1}^{3} c_{2}^{4} b_{1}^{8}+58 A_{1}^{6} c_{2}^{2} b_{1}^{4}+105 A_{1}^{9}\right) a_{2}^{2} \\
& -2 A_{1}^{2} c_{2}^{3}\left(-9 c_{2}^{6} b_{1}^{12}+7 A_{1}^{6} c_{2}^{2} b_{1}^{4}+140 A_{1}^{9}\right) a_{2} \\
& +c_{2}^{4}\left(c_{2}^{8} b_{1}^{16}+4 A_{1}^{3} c_{2}^{6} b_{1}^{12}-4 A_{1}^{6} c_{2}^{4} b_{1}^{8}-12 A_{1}^{9} c_{2}^{2} b_{1}^{4}+70 A_{1}^{12}\right), \\
& p_{11}=b_{1}^{2}\left(-b_{1}^{4} a_{2}^{7}+28 A_{1} b_{1}^{4} c_{2} a_{2}^{6}+6\left(11 A_{1}^{5}+25 b_{1}^{4} c_{2}^{2} A_{1}^{2}\right) a_{2}^{5}\right. \\
& +\left(70 c_{2}^{5} b_{1}^{8}-20 A_{1}^{3} c_{2}^{3} b_{1}^{4}-29 A_{1}^{6} c_{2}\right) a_{2}^{4} \\
& +\left(60 A_{1} c_{2}^{6} b_{1}^{8}-271 A_{1}^{4} c_{2}^{4} b_{1}^{4}-264 A_{1}^{7} c_{2}^{2}\right) a_{2}^{3} \\
& +2\left(153 c_{2}^{3} A_{1}^{8}+42 b_{1}^{4} c_{2}^{5} A_{1}^{5}-55 b_{1}^{8} c_{2}^{7} A_{1}^{2}\right) a_{2}^{2} \\
& +\left(4 c_{2}^{10} b_{1}^{12}+38 A_{1}^{6} c_{2}^{6} b_{1}^{4}-34 A_{1}^{9} c_{2}^{4}\right) a_{2} \\
& \left.+A_{1} c_{2}^{5}\left(2 c_{2}^{6} b_{1}^{12}+15 A_{1}^{3} c_{2}^{4} b_{1}^{8}-8 A_{1}^{6} c_{2}^{2} b_{1}^{4}-45 A_{1}^{9}\right)\right), \\
& p_{12}=6 A_{1}^{2} b_{1}^{4} a_{2}^{6}+\left(49 c_{2}^{3} b_{1}^{8}+96 A_{1}^{3} c_{2} b_{1}^{4}\right) a_{2}^{5}+\left(164 A_{1} c_{2}^{4} b_{1}^{8}+43 A_{1}^{4} c_{2}^{2} b_{1}^{4}+56 A_{1}^{7}\right) a_{2}^{4} \\
& -2\left(112 c_{2} A_{1}^{8}+164 b_{1}^{4} c_{2}^{3} A_{1}^{5}+67 b_{1}^{8} c_{2}^{5} A_{1}^{2}\right) a_{2}^{3} \\
& +\left(3 c_{2}^{8} b_{1}^{12}-56 A_{1}^{3} c_{2}^{6} b_{1}^{8}+96 A_{1}^{6} c_{2}^{4} b_{1}^{4}+336 A_{1}^{9} c_{2}^{2}\right) a_{2}^{2} \\
& +A_{1} c_{2}^{3}\left(12 c_{2}^{6} b_{1}^{12}+33 A_{1}^{3} c_{2}^{4} b_{1}^{8}+104 A_{1}^{6} c_{2}^{2} b_{1}^{4}-224 A_{1}^{9}\right) a_{2} \\
& +A_{1}^{2} c_{2}^{4}\left(6 c_{2}^{6} b_{1}^{12}-17 A_{1}^{6} c_{2}^{2} b_{1}^{4}+56 A_{1}^{9}\right), \\
& p_{13}=b_{1}^{2}\left(c_{2}^{11} b_{1}^{12}+12 A_{1}^{3} c_{2}^{9} b_{1}^{8}+2 A_{1}^{6} c_{2}^{7} b_{1}^{4}-74 A_{1}^{9} c_{2}^{5}\right.
\end{aligned}
$$




$$
\begin{aligned}
& +2 a_{2}^{3} c_{2}^{2}\left(c_{2}^{2} b_{1}^{4}+A_{1}^{3}\right)\left(35 b_{1}^{4} c_{2}^{2}-113 A_{1}^{3}\right) \\
& +5 a_{2}^{5}\left(5 A_{1}^{4}+12 b_{1}^{4} c_{2}^{2} A_{1}\right)+10 a_{2}^{4}\left(5 c_{2} A_{1}^{5}+16 b_{1}^{4} c_{2}^{3} A_{1}^{2}\right) \\
& +2 a_{2}^{2}\left(-2 A_{1} c_{2}^{7} b_{1}^{8}-63 A_{1}^{4} c_{2}^{5} b_{1}^{4}+64 A_{1}^{7} c_{2}^{3}\right) \\
& \left.+a_{2}\left(97 c_{2}^{4} A_{1}^{8}+60 b_{1}^{4} c_{2}^{6} A_{1}^{5}-43 b_{1}^{8} c_{2}^{8} A_{1}^{2}\right)\right) \text {, } \\
& p_{14}=24 A_{1}^{2} b_{1}^{4} c_{2} a_{2}^{5}+2\left(35 c_{2}^{4} b_{1}^{8}+54 A_{1}^{3} c_{2}^{2} b_{1}^{4}+14 A_{1}^{6}\right) a_{2}^{4} \\
& -28\left(-3 A_{1} c_{2}^{5} b_{1}^{8}+5 A_{1}^{4} c_{2}^{3} b_{1}^{4}+4 A_{1}^{7} c_{2}\right) a_{2}^{3} \\
& +6\left(28 c_{2}^{2} A_{1}^{8}-22 b_{1}^{4} c_{2}^{4} A_{1}^{5}-13 b_{1}^{8} c_{2}^{6} A_{1}^{2}\right) a_{2}^{2} \\
& +\left(3 c_{2}^{9} b_{1}^{12}-12 A_{1}^{3} c_{2}^{7} b_{1}^{8}+132 A_{1}^{6} c_{2}^{5} b_{1}^{4}-112 A_{1}^{9} c_{2}^{3}\right) a_{2} \\
& +2 A_{1} c_{2}^{4}\left(2 c_{2}^{6} b_{1}^{12}+3 A_{1}^{3} c_{2}^{4} b_{1}^{8}+4 A_{1}^{6} c_{2}^{2} b_{1}^{4}+14 A_{1}^{9}\right) \text {, } \\
& p_{15}=b_{1}^{2}\left(-A_{1}^{2} b_{1}^{8} c_{2}^{9}+12 A_{1}^{5} b_{1}^{4} c_{2}^{7}-59 A_{1}^{8} c_{2}^{5}+4 a_{2}^{5} A_{1}^{3}+a_{2}^{4}\left(37 c_{2} A_{1}^{4}+80 b_{1}^{4} c_{2}^{3} A_{1}\right)\right. \\
& +a_{2}^{3}\left(70 A_{1}^{2} b_{1}^{4} c_{2}^{4}-76 A_{1}^{5} c_{2}^{2}\right) \\
& \left.-2 a_{2}^{2}\left(-18 c_{2}^{7} b_{1}^{8}+72 A_{1}^{3} c_{2}^{5} b_{1}^{4}+17 A_{1}^{6} c_{2}^{3}\right)+2 a_{2}\left(-7 A_{1} b_{1}^{8} c_{2}^{8}-9 A_{1}^{4} b_{1}^{4} c_{2}^{6}+64 A_{1}^{7} c_{2}^{4}\right)\right) \text {, } \\
& p_{16}=4\left(2 A_{1}^{5}+9 b_{1}^{4} c_{2}^{2} A_{1}^{2}\right) a_{2}^{4}+4 c_{2}\left(14 c_{2}^{4} b_{1}^{8}+7 A_{1}^{3} c_{2}^{2} b_{1}^{4}-8 A_{1}^{6}\right) a_{2}^{3} \\
& +4\left(7 A_{1} c_{2}^{6} b_{1}^{8}-33 A_{1}^{4} c_{2}^{4} b_{1}^{4}+12 A_{1}^{7} c_{2}^{2}\right) a_{2}^{2} \\
& -4\left(8 c_{2}^{3} A_{1}^{8}-9 b_{1}^{4} c_{2}^{5} A_{1}^{5}+7 b_{1}^{8} c_{2}^{7} A_{1}^{2}\right) a_{2}+c_{2}^{4}\left(c_{2}^{6} b_{1}^{12}+32 A_{1}^{6} c_{2}^{2} b_{1}^{4}+8 A_{1}^{9}\right), \\
& p_{17}=b_{1}^{2} c_{2}\left(-2 A_{1} b_{1}^{8} c_{2}^{8}+2 A_{1}^{4} b_{1}^{4} c_{2}^{6}-24 A_{1}^{7} c_{2}^{4}+60 a_{2}^{3} A_{1} b_{1}^{4} c_{2}^{3}+8 a_{2}^{4} A_{1}^{3}\right. \\
& \left.-6 a_{2}^{2}\left(8 c_{2}^{2} A_{1}^{5}+b_{1}^{4} c_{2}^{4} A_{1}^{2}\right)+a_{2}\left(9 c_{2}^{7} b_{1}^{8}-56 A_{1}^{3} c_{2}^{5} b_{1}^{4}+64 A_{1}^{6} c_{2}^{3}\right)\right), \\
& p_{18}=a_{2}^{4} A_{1}^{4}-4 a_{2}^{3} c_{2}\left(A_{1}^{3}-6 b_{1}^{4} c_{2}^{2}\right) A_{1}^{2}+c_{2}^{4}\left(-4 c_{2}^{4} b_{1}^{8}+24 A_{1}^{3} c_{2}^{2} b_{1}^{4}+A_{1}^{6}\right) A_{1}^{2} \\
& -4 a_{2} c_{2}^{3}\left(-c_{2}^{4} b_{1}^{8}+6 A_{1}^{3} c_{2}^{2} b_{1}^{4}+A_{1}^{6}\right) A_{1}+a_{2}^{2}\left(28 c_{2}^{6} b_{1}^{8}-24 A_{1}^{3} c_{2}^{4} b_{1}^{4}+6 A_{1}^{6} c_{2}^{2}\right), \\
& p_{19}=b_{1}^{2} c_{2}^{2}\left(c_{2}^{7} b_{1}^{8}-8 A_{1}^{3} c_{2}^{5} b_{1}^{4}+4 a_{2}^{3} A_{1}^{3}-4 A_{1}^{6} c_{2}^{3}-12 a_{2}^{2} A_{1} c_{2}\left(A_{1}^{3}-2 b_{1}^{4} c_{2}^{2}\right)\right. \\
& \left.+4 a_{2}\left(3 A_{1}^{5} c_{2}^{2}-4 A_{1}^{2} b_{1}^{4} c_{2}^{4}\right)\right) \text {, } \\
& p_{20}=2 b_{1}^{4} c_{2}^{4}\left(3 c_{2}^{2} A_{1}^{4}+3 a_{2}^{2} A_{1}^{2}+a_{2}\left(4 b_{1}^{4} c_{2}^{3}-6 A_{1}^{3} c_{2}\right)\right) \text {, } \\
& p_{21}=4 A_{1} b_{1}^{6} c_{2}^{6}\left(a_{2}-A_{1} c_{2}\right) \text {, } \\
& p_{22}=b_{1}^{8} c_{2}^{8} \text {. }
\end{aligned}
$$

\section{Competing interests}

The authors declare that they have no competing interests.

\section{Authors' contributions}

Each of the authors, VH, MRSK and EP, contributed to each part of this work equally and read and approved the final version of the manuscript.

\section{Author details}

'Division of Mathematics, Faculty of Mechanical Engineering, University of Sarajevo, Sarajevo, Bosnia and Herzegovina. ${ }^{2}$ Department of Mathematics, University of Rhode Island, Kingston, Rhode Island 02881-0816, USA. ${ }^{3}$ Department of Mathematics, University of Sarajevo, Sarajevo, Bosnia and Herzegovina.

\section{Acknowledgements}

The authors are grateful to the three anonymous referees for numerous helpful and constructive suggestions which have improved the presentation of results. 
Received: 26 August 2014 Accepted: 14 November 2014 Published: 01 Dec 2014

\section{References}

1. Garić-Demirović, M, Kulenović, MRS, Nurkanović, M: Global behavior of four competitive rational systems of difference equations in the plane. Discrete Dyn. Nat. Soc. 2009, Article ID 153058 (2009)

2. Kulenović, MRS, Merino, O: Global bifurcation for competitive systems in the plane. Discrete Contin. Dyn. Syst., Ser. B 12, 133-149 (2009)

3. Kulenović, MRS, Merino, O: Invariant manifolds for competitive discrete systems in the plane. Int. J. Bifurc. Chaos Appl. Sci. Eng. 20, 2471-2486 (2010)

4. Burgić, D, Kulenović, MRS, Nurkanović, M: Global dynamics of a rational system of difference equations in the plane. Commun. Appl. Nonlinear Anal. 15, 71-84 (2008)

5. Clark, D, Kulenović, MRS, Selgrade, JF: Global asymptotic behavior of a two dimensional difference equation modelling competition. Nonlinear Anal. TMA 52, 1765-1776 (2003)

6. Cushing, JM, Levarge, S, Chitnis, N, Henson, SM: Some discrete competition models and the competitive exclusion principle. J. Differ. Equ. Appl. 10, 1139-1152 (2004)

7. Hirsch, M, Smith, H: Monotone dynamical systems. In: Handbook of Differential Equations: Ordinary Differential Equations, vol. II, pp. 239-357. Elsevier, Amsterdam (2005)

8. Kulenović, MRS, Merino, O: Discrete Dynamical Systems and Difference Equations with Mathematica. Chapman \& Hall/CRC, Boca Raton (2002)

9. Kulenović, MRS, Merino, O: Competitive-exclusion versus competitive-coexistence for systems in the plane. Discrete Contin. Dyn. Syst., Ser. B 6, 1141-1156 (2006)

10. Kulenović, MRS, Nurkanović, M: Asymptotic behavior of a linear fractional system of difference equations. J. Inequal. Appl. 2005, 127-143 (2005)

11. Leonard, WJ, May, R: Nonlinear aspects of competition between species. SIAM J. Appl. Math. 29, $243-275$ (1975)

12. Smith, HL: Periodic competitive differential equations and the discrete dynamics of competitive maps. J. Differ. Equ. 64, 165-194 (1986)

13. Smith, HL: Periodic solutions of periodic competitive and cooperative systems. SIAM J. Math. Anal. 17, 1289-1318 (1986)

14. Smith, HL: Planar competitive and cooperative difference equations. J. Differ. Equ. Appl. 3, 335-357 (1998)

15. Brett, A, Kulenović, MRS: Basins of attraction of equilibrium points of monotone difference equations. Sarajevo J. Math. 5, 211-233 (2009)

16. Burgić, D, Kalabušić, S, Kulenović, MRS: Nonhyperbolic dynamics for competitive systems in the plane and global period-doubling bifurcations. Adv. Dyn. Syst. Appl. 3, 229-249 (2008)

17. Camouzis, E, Kulenović, MRS, Ladas, G, Merino, O: Rational systems in the plane. J. Differ. Equ. Appl. 15, 303-323 (2009)

18. Clark, D, Kulenović, MRS: On a coupled system of rational difference equations. Comput. Math. Appl. 43, 849-867 (2002)

19. de Mottoni, P, Schiaffino, A: Competition systems with periodic coefficients: a geometric approach. J. Math. Biol. 11, 319-335 (1981)

20. Franke, JE, Yakubu, A-A: Mutual exclusion verses coexistence for discrete competitive systems. J. Math. Biol. 30 , 161-168 (1991)

21. Franke, JE, Yakubu, A-A: Geometry of exclusion principles in discrete systems. J. Math. Anal. Appl. 168, 385-400 (1992)

22. Kalabušić, S, Kulenović, MRS, Pilav, E: Multiple attractors for a competitive system of rational difference equations in the plane. Abstr. Appl. Anal. 2011, Article ID 295308 (2011)

23. Kalabušić, S, Kulenović, MRS, Pilav, E: Dynamics of a two-dimensional system of rational difference equations of Leslie-Gower type. Adv. Differ. Equ. 2011, Article ID 29 (2011)

24. Kulenović, MRS, Ladas, G: Dynamics of Second Order Rational Difference Equations with Open Problems and Conjectures. Chapman \& Hall/CRC, Boca Raton (2001)

25. Hess, P: Periodic-Parabolic Boundary Value Problems and Positivity. Pitman Research Notes in Mathematics Series, vol. 247, viii+139 pp. Longman Scientific \& Technical, Harlow (1991)

26. Yang, L, Hou, X, Zeng, Z: Complete discrimination system for polynomials. Sci. China Ser. E 39(6), 628-646 (1996)

27. Basu, S, Merino, O: On the behavior of solutions of a system of difference equations. Commun. Appl. Nonlinear Anal. 16(1), 89-101 (2009)

28. Walker, RJ: Algebraic Curves. Princeton University Press, Princeton (1950)

29. Gelfand, IM, Kapranov, MM, Zelevinsky, AV: Discriminants, Resultants and Multidimensional Determinants. Birkhäuser, Boston (1994)

10.1186/1687-1847-2014-301

Cite this article as: Hadžiabdić et al.: Dynamics of a two-dimensional competitive system of rational difference equations with quadratic terms. Advances in Difference Equations 2014, 2014:301 